\title{
Resolving discharge parameters from atomic oxygen emission
}

Citation for published version (APA):

Viegas, P., Vialetto, L., van de Steeg, A. W., Wolf, A. J., Bongers, W. A., van Rooij, G. J., van de Sanden, M. C. M., Diomede, P., \& Peeters, F. J. J. (2021). Resolving discharge parameters from atomic oxygen emission. Plasma Sources Science \& Technology, 30(6), [065022]. https://doi.org/10.1088/13616595/ac04bd

Document status and date:

Published: 01/06/2021

DOI:

10.1088/1361-6595/ac04bd

Document Version:

Publisher's PDF, also known as Version of record

Document license:

Taverne

Please check the document version of this publication:

- A submitted manuscript is the version of the article upon submission and before peer-review. There can be important differences between the submitted version and the official published version of record.

People interested in the research are advised to contact the author for the final version of the publication, or visit the DOI to the publisher's website.

- The final author version and the galley proof are versions of the publication after peer review.

- The final published version features the final layout of the paper including the volume, issue and page numbers.

Link to publication

\footnotetext{
General rights Owners
rights.

- You may freely distribute the URL identifying the publication in the public portal. please follow below link for the End User Agreement:

www.umlib.nl/taverne-license

Take down policy

If you believe that this document breaches copyright please contact us at:

repository@maastrichtuniversity.nl

providing details and we will investigate your claim.
}

Copyright and moral rights for the publications made accessible in the public portal are retained by the authors and/or other copyright owners and it is a condition of accessing publications that users recognise and abide by the legal requirements associated with these

- Users may download and print one copy of any publication from the public portal for the purpose of private study or research.

- You may not further distribute the material or use it for any profit-making activity or commercial gain

If the publication is distributed under the terms of Article $25 \mathrm{fa}$ of the Dutch Copyright Act, indicated by the "Taverne" license above, 


\title{
Resolving discharge parameters from atomic oxygen emission
}

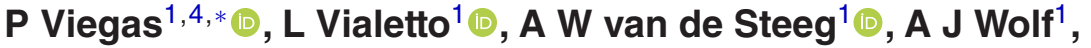 \\ W A Bongers ${ }^{1}$, G J van Rooij ${ }^{1,2,3}{ }^{10}$, M C M van de Sanden ${ }^{1,2}{ }^{-}$, \\ P Diomede ${ }^{1,3}$ and $\mathbf{F}$ J J Peeters ${ }^{1}[0$ \\ ${ }^{1}$ DIFFER—Dutch Institute for Fundamental Energy Research, 5612 AJ Eindhoven, The Netherlands \\ 2 Department of Applied Physics, Eindhoven University of Technology, PO Box 513, 5600 MB \\ Eindhoven, The Netherlands \\ ${ }^{3}$ Faculty of Science and Engineering, Maastricht University, Zwingelput 4, ZW4, $6211 \mathrm{KH}$ Maastricht, \\ The Netherlands \\ E-mail: viegas@mail.muni.cz
}

Received 5 March 2021, revised 6 May 2021

Accepted for publication 25 May 2021

Published 25 June 2021

\begin{abstract}
A method is proposed to spatially resolve discharge parameters from experimental measurements of emission intensity and 1D numerical simulations including an $\mathrm{O}$ atom collisional-radiative model. The method can be used for different plasmas and conditions. Here, contracted microwave discharges for $\mathrm{CO}_{2}$ conversion are studied at intermediate to high pressures (100-300 mbar). Radial profiles of electron density $\left(n_{\mathrm{e}}\right)$ are used as input in the model and corrected to successfully simulate the measured Gaussian profiles of emission intensity of the $777 \mathrm{~nm}$ transition $\left(I_{777}\right)$. As a result, radially-resolved parameters inaccessible in experiments, such as $n_{\mathrm{e}}$, power density $\left(P_{\mathrm{abs}}\right)$, electron temperature $\left(T_{\mathrm{e}}\right)$, electric field and reaction rates, are numerically-obtained for several conditions. $n_{\mathrm{e}}$ and $P_{\mathrm{abs}}$ approximately follow Gaussian profiles that are broader than that of $I_{777}$. For pressures below $150 \mathrm{mbar}$, the difference in full width at half maximum is typically a factor 1.6. This consists in a phenomenon of optical contraction, which is due to concave profiles of $\mathrm{O}$ molar fraction and $T_{\mathrm{e}}$. The implications of the simulated profiles on the study of plasmas for $\mathrm{CO}_{2}$ conversion are discussed and it is shown that these profiles allow to explain high reactor performances at low pressures.
\end{abstract}

Keywords: atomic oxygen kinetics, discharge spatial resolution, optical contraction, discharge contraction, $\mathrm{CO}_{2}$ conversion

(Some figures may appear in colour only in the online journal)

\section{Introduction}

The understanding of low-temperature plasma physics and the optimisation of its applications is dependent on our knowledge of the spatial distributions of physical parameters in discharge reactors. Among those parameters, electron density $\left(n_{\mathrm{e}}\right)$, electron temperature $\left(T_{\mathrm{e}}\right)$, gas composition, gas temperature $\left(T_{\mathrm{g}}\right)$, reduced electric field $\left(E / n_{\mathrm{g}}\right)$, emission intensity

\footnotetext{
* Author to whom any correspondences should be addressed.

${ }^{4}$ Present address: Department of Physical Electronics at the Faculty of Science, Masaryk University, Kotlârská 267/2, 61137 Brno, Czech Republic.
}

and power density $\left(P_{\text {abs }}\right)$ are often some of the most important. In fact, the spatial distribution of discharge parameters has been vastly studied but is only well established for specific discharge configurations. A well known example is the case in low pressure discharge regimes controlled by free diffusion or by ambipolar diffusion, where the radial distribution of $n_{\mathrm{e}}$ in a long cylindrical discharge follows a paraboloidal or a zero-order Bessel function of the first kind with zero at the cylinder wall (Schottky 1924, Parker 1963, Ikegami 1968, Durandet et al 1989, Lieberman and Lichtenberg 2005, Fridman and Kennedy 2004, Moisan and Pelletier 2012). However, 
charged-particle balance in intermediate (a few to hundreds of mbar) to high pressure discharges relies on molecular ion recombination, and thus the Bessel profile cannot be assumed for cylindrical discharges in those conditions.

In several numerical and experimental works on glow and microwave (MW) discharges with atomic and molecular gases, $n_{\mathrm{e}}$ and emission intensity have been shown to have Bessel radial profiles at low pressures and currents when the discharge is diffuse, but significantly different, seemingly Gaussian profiles, at higher pressures or currents when the discharge contracts (Petrov and Ferreira 1999, Martinez et al 2004, Kabouzi et al 2007, Dyatko et al 2008, Golubovskii et al 2011, Shneider et al 2014, Golubovskii et al 2017, Ridenti et al 2018, Zhong et al 2019). In particular, in the work of Dyatko et al (2008) with a 1D-radial model describing an argon glow discharge at intermediate pressure, the ratio between the peak and the average electron densities has been calculated to change from 2.3 for a Bessel profile to 720 for a highly contracted discharge. Then, in Carbone et al (2012), radially-resolved $n_{\mathrm{e}}$ measurements through Thomson scattering in an MW argon plasma at intermediate pressure have been fitted with a Bessel function for which the boundary radius $R$ defined by $n_{\mathrm{e}}(R)=0$ is a free parameter that decreases with pressure. Furthermore, in the 1D models in Gregório et al $(2010,2012)$ of argon MW microplasmas at atmospheric pressure, $n_{\mathrm{e}}$ contracts to a concave shape that is neither Gaussian nor Bessel. On the contrary, in the 2D numerical work in Baeva et al (2018) in an argon plasma torch at atmospheric pressure, the radial profile of $n_{\mathrm{e}}$ presented is rather flat.

Radiative emission takes place naturally in reactive plasmas, and therefore emission intensity can be an easily accessible source of spatially-resolved information in the discharge. Nevertheless, in contracted conditions, the $n_{\mathrm{e}}$ profile can be spatially more extended than the optical appearance of the plasma suggests. Indeed, experimental radial distributions of line emission have been reported to be more compressed than that of $n_{\mathrm{e}}$ (obtained from the Bremsstrahlung continuum) in neon in Golubovskii et al (2011) and in helium in Golubovskii et al (2020). This effect has been called optical contraction and has been attributed in Golubovskii et al (2011) to the influence of electron-electron collisions on the electron energy distribution function (EEDF), which enhances electron-impact excitation and ionisation reactions in the centre of the discharge. However, in Golubovskii et al (2020), this effect seems to be due to the low values of $E / n_{\mathrm{g}}$ in the radial edges, as $E / n_{\mathrm{g}}$ is theoretically estimated to have a significantly concave distribution in that work. The concave shape of $E / n_{\mathrm{g}}$ is in agreement with the experimental measurements and simulations of concave profiles of $T_{\mathrm{e}}$ in Kabouzi et al (2007), Gregório et al (2010), Golubovskii et al (2011), Gregório et al (2012), Golubovskii et al (2017), Ridenti et al (2018), Zhong et al (2019). In fact, the simulations in Ridenti et al (2018) have shown that $T_{\mathrm{e}}$ in Thomson scattering measurements, such as those in van Gessel et al (2012), Carbone et al (2012), has a convex radial profile due to the Maxwellian assumption of this diagnostic, while $T_{\mathrm{e}}$ obtained from the calculated EEDF (2/3 of mean electron energy) actually has a concave radial profile, as does $E / n_{\mathrm{g}}$. Conversely, the $T_{\mathrm{e}}$ and $E / n_{\mathrm{g}}$ radial profiles obtained in the 1D and 2D simulations in Martinez et al (2004), JimenezDiaz et al (2012), Georgieva et al (2017), Baeva et al (2018) have been reported as approximately radially homogeneous. We can conclude that the relationships between the spatial profiles of different discharge parameters so far are not completely understood and cannot be generalised.

In this work, we address the spatial distribution of discharge parameters in $\mathrm{MW} \mathrm{CO}_{2}$ plasma reactors. These are intended to convert greenhouse gas $\mathrm{CO}_{2}$ into carbon-neutral fuels or useful chemicals (Goede et al 2014, Guerra et al 2017, Snoeckx and Bogaerts 2017, Vermeiren and Bogaerts 2020). Spatial distributions in these discharges are particularly interesting, as the discharge radius has been shown to have a strong correlation with reactor performance, with the best conversion and energy efficiencies having been obtained when the core of the discharge has a radially-contracted structure (Fridman 2008, van Rooij et al 2015, den Harder et al 2017, Bongers et al 2017). In particular, the relationship between the spatial distribution of $P_{\text {abs }}$ and the reactor performance has been highlighted in van den Bekerom et al (2019).

Recent studies have addressed the contraction dynamics and the radial structure of MW discharges for $\mathrm{CO}_{2}$ conversion (Wolf et al 2019, Groen et al 2019, Wolf et al 2020a, 2020b, Viegas et al 2020, D'Isa et al 2020). The contraction of a vortex-stabilised $\mathrm{CO}_{2} \mathrm{MW}$ plasma has been characterised in Wolf et al (2019) in relation to its dielectric properties. Then, in Wolf et al (2020b), two distinct contracted discharge modes have been identified and described at pressures above 85 mbar: a low-confinement L-mode at lower pressures and at temperatures between 3000 and $5000 \mathrm{~K}$ and a high-confinement $\mathrm{H}$ mode at higher pressures and at temperatures above $5500 \mathrm{~K}$. The works in Viegas et al (2020) and Wolf et al (2020a) have investigated the contracted modes numerically. The distinct changes in spatial profiles of $n_{\mathrm{e}}$ and $P_{\mathrm{abs}}$ with discharge modes have been shown to be important to obtain accurate descriptions of the reactivity within the plasma, that determines the spatial structure of the plasma itself (Viegas et al 2020), as well as of the follow-up reactions of plasma products in its periphery, that influence the reactor performance (Wolf et al 2020a). Despite the importance of accurately determining these spatial profiles, discharge parameters are difficult to measure with $\mathrm{mm}$ resolution in $\mathrm{CO}_{2} \mathrm{MW}$ plasmas and are often based on the spatial distribution of parameters that are easier to obtain experimentally, such as the radiative emission intensity. As such, the works on $\mathrm{CO}_{2}$ discharge contraction so far have relied on unverified simplifying assumptions on the spatial profiles of discharge parameters, such as $n_{\mathrm{e}}, P_{\mathrm{abs}}$ and $E / n_{\mathrm{g}}$. To selfconsistently describe the relationships between these parameters and improve profile determination, it is very relevant to assess the assumptions used so far.

In this work, numerical simulations are used to determine spatially-resolved discharge parameters in a $\mathrm{CO}_{2} \mathrm{MW}$ discharge from the experimentally measured intensity distribution of the $777 \mathrm{~nm}$ spectral line emission of atomic oxygen: $\mathrm{O}\left(3 \mathrm{p}{ }^{5} \mathrm{P}\right) \rightarrow \mathrm{O}\left(3 \mathrm{~s}^{5} \mathrm{~S}^{0}\right)\left(I_{777}\right)$. This study requires a collisionalradiative model (CRM) of $\mathrm{O}$ atom kinetics. That type of description has been developed and used in works such as 
Dagdigian et al (1988), Stancu et al (2016), Caplinger and Perram (2020), Fiebrandt et al (2020). In particular, in Fiebrandt et al (2020) a CRM has been developed to calculate excited state densities of atomic oxygen in zero dimensions in low pressure plasmas with varying $\mathrm{Ar} / \mathrm{O}_{2}$ mixtures. That work has highlighted the influence of radiative cascading between excited states and of radiation self-absorption. Moreover, in Caplinger and Perram (2020) a CRM focussed on the $777 \mathrm{~nm} / 844 \mathrm{~nm}$ line ratio of atomic oxygen has been developed in zero dimensions to study oxygen plasmas at different pressures and with different dissociation degrees (ratio between $\mathrm{O}$ and $\mathrm{O}_{2}$ ). The results of that CRM have been compared with those of more commonly-used models, such as the extended corona model. The relevance of stepwise excitation and cascade emission has been put forward in Caplinger and Perram (2020). Both of these recent works have discussed the choice and use of kinetic data in their CRMs.

This paper employs for the first time a CRM for atomic oxygen developed in the context of reactive $\mathrm{CO}_{2}$ mixtures at intermediate to high pressures (100-300 mbar). We use it in $1 \mathrm{D}$ to radially resolve $n_{\mathrm{e}}, T_{\mathrm{e}}, E / n_{\mathrm{g}}$ and $P_{\mathrm{abs}}$ in contracted MW discharges for $\mathrm{CO}_{2}$ conversion. In section 2, the experimental set-up is presented, along with the plasma conditions and the measurements undertaken. The set of hypotheses taken in the interpretation of experiments to relate the experimentallymeasured Gaussian profiles $I_{777}(r)$ to the other discharge parameters is also explained. Then, the 1D-radial model developed in this work is explained in section 3, including the CRM for atomic oxygen. The simulation results are presented in section 4 for the different sets of conditions presented in section 2 . First, an $n_{\mathrm{e}}(r)$ profile calculated directly from experimental data is used as input in the simulations, in order to illustrate the shortcomings of some of the hypotheses taken. The dependence of $n_{\mathrm{e}}(r)$ on $I_{777}(r)$, and vice-versa, is obtained and explained from the simulations, and leads to correcting the hypotheses considered so far and the assumptions for $n_{\mathrm{e}}(r)$. The simulations show that the corrected assumptions are consistent with the measurements, and thus provide radiallyresolved discharge parameters. Finally, the implications of these findings on the study of $\mathrm{MW}$ discharges for $\mathrm{CO}_{2}$ conversion are demonstrated and discussed, along with the possibilities to apply this correction procedure to relate discharge parameters in different plasmas.

\section{Experimental set-up, hypotheses and conditions}

\subsection{Experimental set-up and hypotheses on plasma parameters}

The discharges studied in this work are obtained experimentally in a vortex-stabilised MW reactor. This configuration has been used in several works on plasma-driven $\mathrm{CO}_{2}$ conversion (Butylkin et al 1981, Fridman 2008, Bongers et al 2017). The set-up is presented in figure 1 and described in more detail in Wolf et al (2019). It consists of a $2.45 \mathrm{GHz}$ MW source, a rectangular waveguide field applicator and a three-stub tuner for impedance matching. The electromagnetic

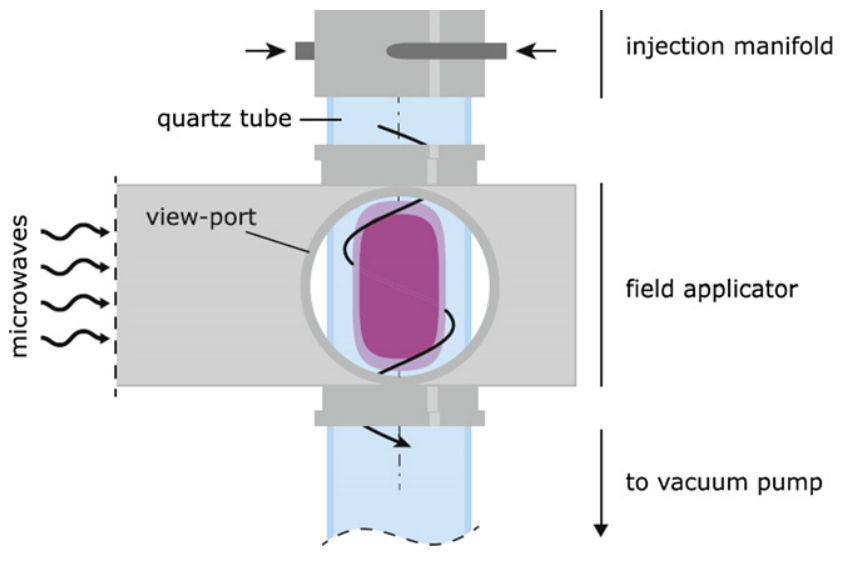

Figure 1. Illustration of the experimental setup, including the main plasma reactor components. Reproduced from [Wolf et al [2020b]]. ๑ IOP Publishing Ltd. All rights reserved.

(EM) field is continuously coupled to a $\mathrm{CO}_{2}$ gas flow, which is contained in a quartz tube of inner radius $R_{\text {tube }}=13.5 \mathrm{~mm}$. The wave electric field is directed parallel to the discharge tube (transverse-electric $\left(\mathrm{TE}_{10}\right)$ single mode standing wave). The plasma is stabilised in the centre of the tube by means of a vortex flow, achieved by tangential gas injection, to prevent plasma-induced wall damage, as in Fleisch et al (2007). The discharge characteristics and the related performance of $\mathrm{CO}_{2}$ conversion to $\mathrm{CO}$ in this reactor have been studied in detail in recent years in Wolf et al (2019, 2020b), Pietanza et al (2020), Wolf et al (2020a), Viegas et al (2020).

In Wolf et al (2019), the intensity distribution of the 777 $\mathrm{nm}$ spectral line emission of atomic oxygen $\left(\mathrm{O}\left(3 \mathrm{p}{ }^{5} \mathrm{P}\right) \rightarrow\right.$ $\left.\mathrm{O}\left(3 \mathrm{~s}{ }^{5} \mathrm{~S}^{0}\right)\right)$ has been measured to have a Gaussian radial profile $I_{777}(r)$ with full width at half maximum (FWHM) $\Lambda_{777}$ and characteristic width (or standard deviation) $\sigma_{777}=$ $\Lambda_{777} /(2 \sqrt{2 \ln (2)})$, that are invariant under an Abel transform:

$$
I_{777}(r)=I_{777}(0) \exp \left(-\frac{1}{2} \frac{r^{2}}{\sigma_{777}^{2}}\right) .
$$

It is assumed in Wolf et al (2019) that $n_{\mathrm{e}}(r)$ also follows a Gaussian radial profile, with peak $n_{\mathrm{e} 0}$, and is proportional to $I_{777}(r)$, with a proportionality parameter $s$, such that:

$$
n_{\mathrm{e}}(r)=n_{\mathrm{e} 0} \exp \left(-\frac{1}{2} \frac{r^{2}}{\left(\sqrt{s} \sigma_{777}\right)^{2}}\right), \quad 1 \leqslant s \leqslant 2 .
$$

That assumption is based on the following hypotheses:

(1) The upper state of the $777 \mathrm{~nm}$ emission, $\mathrm{O}\left(3 \mathrm{p}{ }^{5} \mathrm{P}\right)$, of density $n_{\mathrm{O}\left(3 \mathrm{p}^{5} \mathrm{P}\right)}$, is produced mostly by electron-impact excitation of the atomic oxygen ground-state $\mathrm{O}\left(2 \mathrm{p}^{4}{ }^{3} \mathrm{P}\right)$, of density $n_{\mathrm{O}\left(2 \mathrm{p}^{4} \mathrm{P}\right)}$, with rate coefficient $k_{\mathrm{exc}}\left(T_{\mathrm{e}}\right) . \mathrm{O}\left(3 \mathrm{p}{ }^{5} \mathrm{P}\right)$ is mostly destroyed by radiative emission with coefficient $\tau_{\text {rad }}^{-1}$ and quenching by any neutral species (with gas density $n_{\mathrm{g}}$ ) with coefficient $k_{\mathrm{Q}}$. The radiation balance is then expressed as:

$$
I_{777}=\tau_{\text {rad }}^{-1} n_{\mathrm{O}\left(3 \mathrm{p}^{5} \mathrm{P}\right)} \simeq \tau_{\mathrm{rad}}^{-1} \frac{n_{\mathrm{e}} n_{\mathrm{O}\left(2 \mathrm{p}^{4}{ }^{3} \mathrm{P}\right)} k_{\mathrm{exc}}\left(T_{\mathrm{e}}\right)}{\tau_{\mathrm{rad}}^{-1}+n_{\mathrm{g}} k_{\mathrm{Q}}} .
$$


(2) $\tau_{\text {rad }}^{-1}$ and $k_{\mathrm{Q}}$ are radially homogeneous, and $n_{\mathrm{g}} k_{\mathrm{Q}}>\tau_{\text {rad }}^{-1}$. Thus:

$$
I_{777} \propto n_{\mathrm{e}} x_{\mathrm{O}} k_{\mathrm{exc}}\left(T_{\mathrm{e}}\right)
$$

where $x_{\mathrm{O}}=n_{\mathrm{O}\left(2 \mathrm{p}^{4}{ }^{3} \mathrm{P}\right)} / n_{\mathrm{g}}$.

(3) $E / n_{\mathrm{g}}$ and $T_{\mathrm{e}}$ are radially homogeneous in the plasma region, and so is $k_{\text {exc }}\left(T_{\mathrm{e}}\right)$.

(4) $x_{\mathrm{O}}$ is defined by heavy-particle thermal reactions that are independent of $n_{\mathrm{e}}$, and by electron-impact reactions with rates proportional to $n_{\mathrm{e}}$. It is assumed that:

$$
I_{777} \propto n_{\mathrm{e}}^{s}, \quad 1 \leqslant s \leqslant 2 .
$$

The line-integrated electron density at the axial centre of the discharge $\left(n_{\mathrm{e} \Lambda}\right)$ has been measured in Wolf et al (2019), and thus the peak of $n_{\mathrm{e}}$ and the plasma diameter $\Lambda_{n_{\mathrm{e}}}$ (FWHM of the $n_{\mathrm{e}}(r)$ profile) have been calculated according to the assumption in equation (2) as function of $s$ :

$$
\begin{gathered}
n_{\mathrm{e} 0}=n_{\mathrm{e} \Lambda} / \sqrt{2 \pi\left(\sqrt{s} \sigma_{777}\right)^{2}} \\
\Lambda_{n_{\mathrm{e}}}=\sqrt{s} \Lambda_{777} .
\end{gathered}
$$

Using these hypotheses, in Wolf et al (2019) the radius of the discharge column and the skin-depth of wave absorption have been found to have approximately the same value, for $1 \leqslant s \leqslant 2$. Then, in the subsequent studies of this discharge (Groen et al 2019, Wolf et al 2020b, Pietanza et al 2020, Wolf et al 2020a, Viegas et al 2020), as well as in the investigation of a similar $\mathrm{CO}_{2} \mathrm{MW}$ discharge (D'Isa et al 2020), the assumption in equation (2) has been used, with additional hypotheses:

(5) $x_{\mathrm{O}}$ is defined by thermal chemistry and the radial gradient of gas temperature is lower than the one of $n_{\mathrm{e}}$. Hence, $x_{\mathrm{O}}$ is radially homogeneous in the plasma region, and therefore:

$$
s=1 ; \quad n_{\mathrm{e}}(r)=n_{\mathrm{e} 0} \exp \left(-\frac{1}{2} \frac{r^{2}}{\sigma_{777}^{2}}\right) .
$$

(6) As the temperature, composition, $E / n_{\mathrm{g}}, T_{\mathrm{e}}$ and electron collision frequency $\nu_{\mathrm{c}}$ are considered radially homogeneous, the MW absorbed power density $\left(P_{\mathrm{abs}}\right)$ is assumed to have the same radial profile as $n_{\mathrm{e}}$ (Wolf et al 2020b):

$$
P_{\mathrm{abs}}(r)=P_{\mathrm{abs} 0} \exp \left(-\frac{1}{2} \frac{r^{2}}{\sigma_{777}^{2}}\right),
$$

where $P_{\mathrm{abs} 0}$ is obtained by dividing the total input power by the normalised volume integral of the emission intensity.

\subsection{Experimental conditions}

In this paper, we use the model described in section 3 to evaluate the hypotheses listed in section 2.1 and to spatially resolve discharge parameters. Here we describe the different sets of experimental conditions considered as input in the model.

2.2.1. Condition A: low-confinement discharge. Firstly, a discharge in low-confinement mode, according to the definition in Wolf et al (2020b), is studied. This is a well characterised discharge, which is contracted but with $\Lambda_{777}$ close to half the tube diameter. It has been obtained at 110 mbar with a fixed steady-state input power of $950 \mathrm{~W}$ and a flow rate of $9 \mathrm{slm}$. The Gaussian-shaped emission intensity of the $777 \mathrm{~nm}$ line (following equation (1)) has a radial FWHM $\Lambda_{777}=5.88 \mathrm{~mm}$ and an axial FWHM $L_{777}=21.40 \mathrm{~mm}$. The line-integrated electron density $n_{\mathrm{e} \Lambda}$ has been measured through $140 \mathrm{GHz}$ MW interferometry and the profile $n_{\mathrm{e}}(r)$ has been assumed according to equations (6)-(8). The relative uncertainty in the measurement of $n_{\mathrm{e} \Lambda}$ is of the order of $100 \%$ for pressures below 110 mbar and then decreases with pressure, reaching values of the order of $10 \%$ for pressures above 200 mbar (Wolf et al 2019, Viegas et al 2020). This diagnostic considers the plasma as an infinite homogeneous slab, which applies when the plasma size is large compared to the diagnostic beam dimensions. Since this approximation collapses under the narrow plasma conditions considered in this work, a correction factor is applied which accounts for the partial pass-through of the diagnostic beam through the plasma medium. This correction has not been considered in previous works (Wolf et al 2019, Groen et al 2019, Viegas et al 2020), which might lead to an underestimation of $n_{\mathrm{e}}$ in those works. The partial pass-through effect is accounted for by applying a correction factor $\alpha$, whose calculation is further described in Mousavi et al (2021), such that:

$$
n_{\mathrm{e} \Lambda \text {, corrected }}=n_{\mathrm{e} \Lambda \text {,measured }} \times \alpha \text {. }
$$

The correction factor $\alpha$ is inversely proportional to the interferometry frequency and to $\Lambda_{777}$. In the current case, $\alpha=1.32$ and the corrected $n_{\mathrm{e} 0}=9.11 \times 10^{18} \mathrm{~m}^{-3}$. The rotational temperature (taken as the same as the translational temperature $T_{\mathrm{g}}$ ) and the densities of the main species in the plasma reactor $\left(\mathrm{CO}_{2}, \mathrm{CO}, \mathrm{O}_{2}\right.$ and $\left.\mathrm{O}\right)$ are measured locally at the axial position of the centre of the waveguide with $1 \mathrm{~mm}$ radial resolution through spontaneous rotational Raman scattering. This diagnostic has been used to measure temperature in van den Bekerom et al (2019, 2020), van de Steeg et al (2020) and is further explained in van de Steeg et al (2021). As pointed out in our previous works assessing measurements and simulations of the main neutral species densities (Wolf et al 2020a, Viegas et al 2020, van de Steeg et al 2021), their value and spatial distribution are determined mostly by thermally-driven neutral chemistry and by fast transport processes. The data taken as input in the model are presented in figure 2. On the left side of the figure, the assumed profile $n_{\mathrm{e}}(r)$ and the measured profile $T_{\mathrm{g}}(r)$, and on the right side, the measured molar fractions of the main species.

2.2.2. Condition B: high-confinement discharge. The main characteristics of the studied discharges are defined by their confinement degree, which is largely dependent on input power and pressure (Wolf et al 2020b). As such, it is important to evaluate discharge parameters also in high-confinement mode. A flow rate of $12 \mathrm{slm}$ is used, and a high-confinement discharge is obtained at 150 mbar with an input MW power of $860 \mathrm{~W} . T_{\mathrm{g}}$ and composition are measured through Raman scattering, $n_{\mathrm{e} \Lambda}$ is measured through $140 \mathrm{GHz}$ interferometry and $n_{\mathrm{e}}(r)$ is assumed through equation (8) after considering 

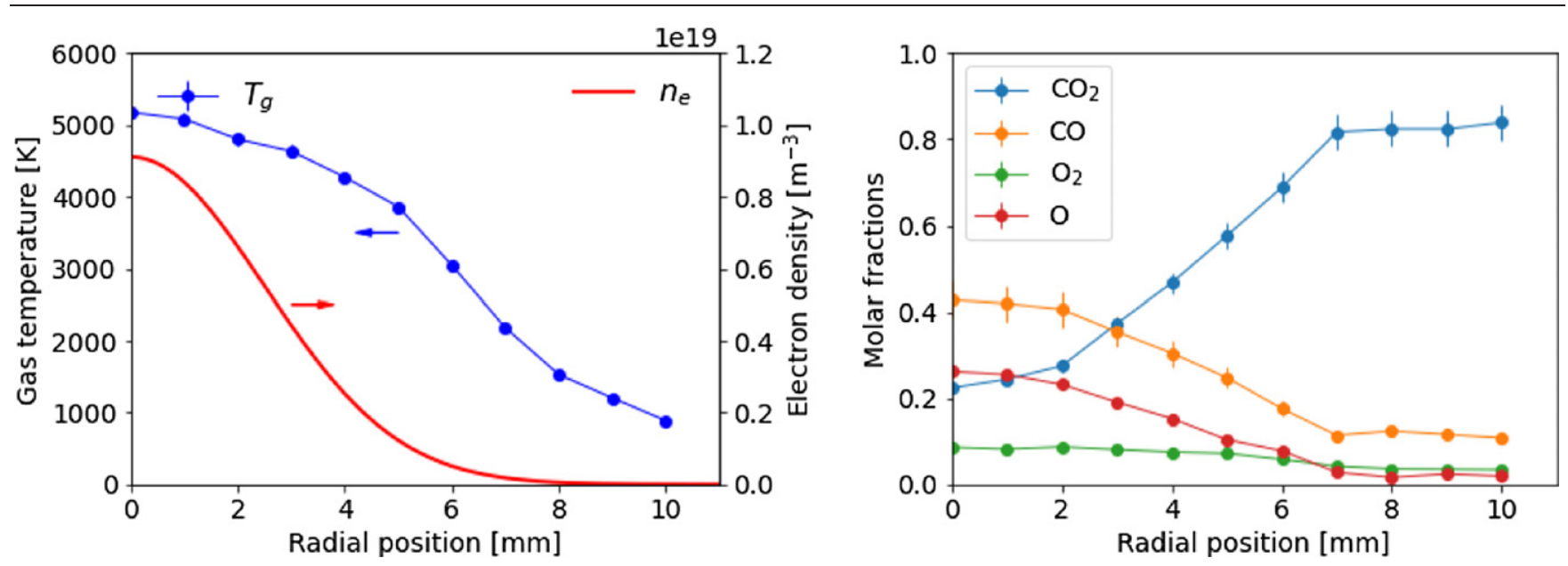

Figure 2. Radial profiles of experimental input parameters in condition $\mathrm{A}(950 \mathrm{~W}, 9 \mathrm{slm}$ and $110 \mathrm{mbar})$. On the left, the assumed $n_{\mathrm{e}}(r)$ and the measured $T_{\mathrm{g}}(r)$. On the right, the measured molar fractions of $\mathrm{CO}_{2}, \mathrm{CO}, \mathrm{O}_{2}$ and $\mathrm{O}$.
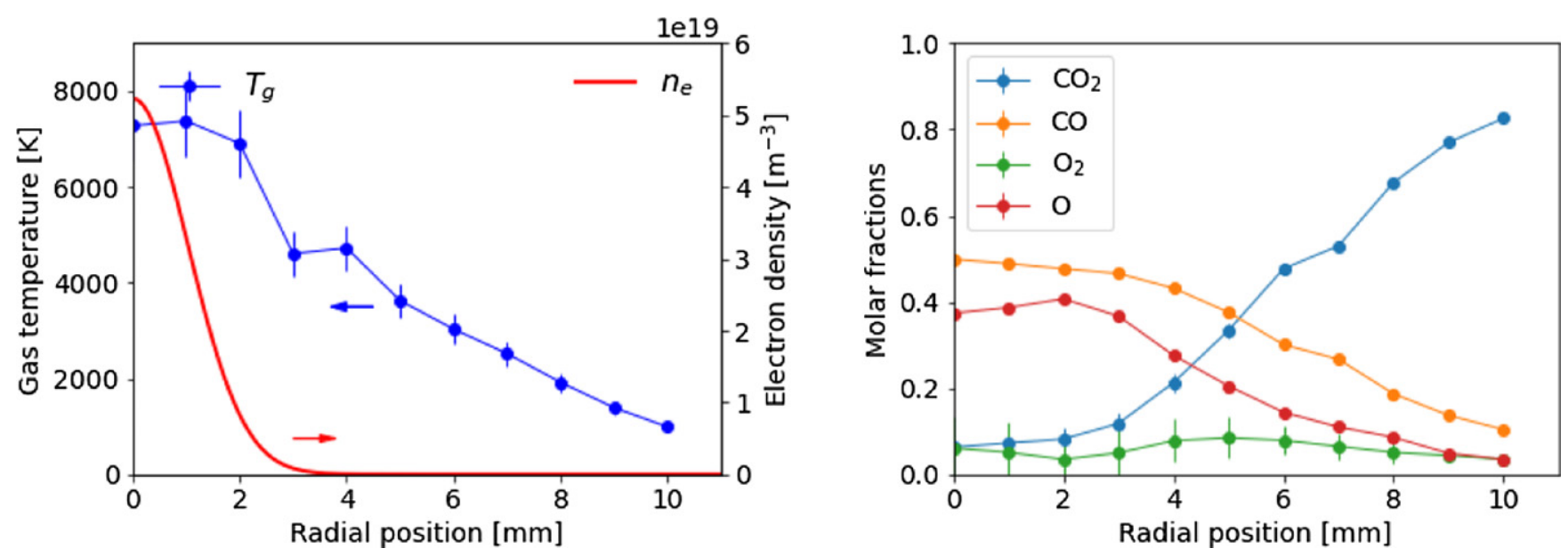

Figure 3. Radial profiles of experimental input parameters in condition $\mathrm{B}(860 \mathrm{~W}, 12 \mathrm{slm}$ and $150 \mathrm{mbar})$. On the left, the assumed $n_{\mathrm{e}}(r)$ and the measured $T_{\mathrm{g}}(r)$. On the right, the measured molar fractions of $\mathrm{CO}_{2}, \mathrm{CO}, \mathrm{O}_{2}$ and $\mathrm{O}$.

the correction factor $\alpha$, as for condition A. The length $L_{777}$ is $26.71 \mathrm{~mm}$, the diameter $\Lambda_{777}$ is $2.46 \mathrm{~mm}$, below half of that of condition A, and the correction factor $\alpha$ is 2.69. $n_{\mathrm{e}}(r)$ and $T_{\mathrm{g}}(r)$ are represented on the left side of figure 3 . The measured composition is shown on the right side of the same figure. The $n_{\mathrm{e}}(r)$ and $T_{\mathrm{g}}(r)$ profiles clearly have sharper gradients than in condition $\mathrm{A}$ and, as a result of the higher temperature, the $\mathrm{CO}$ and $\mathrm{O}$ molar fractions are higher and have broader profiles than in condition A.

2.2.3. Set of conditions $C$ : input from flow modelling. In some high-confinement conditions, emission from $\mathrm{C}_{2}$ is present in the plasma (although $\mathrm{C}_{2}$ remains a minor constituent), which severely cripples the Raman scattering diagnostic. Therefore, it is relevant to study spatial profiles of discharge parameters in cases where neutral composition is known from simulation results, which also allows wider parameter scans. Unlike the case in conditions $\mathrm{A}$ and $\mathrm{B}$, in the set of conditions $\mathrm{C}$ we take the radial distributions of $T_{\mathrm{g}}$ and neutral composition from the simulation results of the 2D model in Wolf et al (2020a), at the axial position of maximum temperature. In that work, the peak gas temperature in the centre of the discharge has been taken as input from Doppler broadening measurements of the $777 \mathrm{~nm}$ atomic oxygen line emission (Wolf et al 2019). We study 7 discharge conditions in different confinement modes by changing only pressure. They are obtained with $1400 \mathrm{~W}$ input power and $18 \mathrm{slm}$ flow rate and the pressure is increased from 108 mbar to 296 mbar. These conditions are described in Wolf et al (2019) and in Viegas et al (2020). However, the plasma dimensions considered here have been found as in Wolf et al (2020a), in a slightly different way than in the previous works. $n_{\mathrm{e}}(r)$ is obtained from equation (8), with $n_{\mathrm{e} \Lambda}$ measured from $168 \mathrm{GHz}$ interferometry. Although $n_{\mathrm{e} 0}$ is displayed in Wolf et al (2019) and in Viegas et al (2020), the correction factor $\alpha$ is not taken into account in the values in those works. For the sake of brevity, the radial profiles of $n_{\mathrm{e}}, T_{\mathrm{g}}$ and composition are not represented here, but $T_{\mathrm{g}}(r)$ and the molar fraction $x_{\mathrm{O}}(r)$ are shown later in figure 13. Moreover, some of the main plasma parameters are listed in table 1 . It can be noticed from $\Lambda_{777}$ in table 1 that the confinement degree of these discharges (except at 108 mbar) stands between the ones of conditions A 
Table 1. Experimental input parameters in the set of conditions $\mathrm{C}$ : pressure, length, diameter, $n_{\mathrm{e}}$ correction factor, corrected $n_{\mathrm{e}}$ peak and maximum of $T_{\mathrm{g}}$.

\begin{tabular}{lccccc}
\hline$p$ (mbar) & $L_{777}(\mathrm{~mm})$ & $\Lambda_{777}(\mathrm{~mm})$ & $\alpha$ & $n_{\mathrm{e} 0}\left(10^{18} \mathrm{~m}^{-3}\right)$ & $T_{\mathrm{g}, \max }(\mathrm{K})$ \\
\hline 108 & 12.1 & 6.44 & 1.09 & 1.61 & 4927 \\
121 & 15.4 & 4.77 & 1.25 & 8.24 & 5562 \\
141 & 18.9 & 3.97 & 1.40 & 24.62 & 6082 \\
161 & 22.5 & 3.68 & 1.47 & 26.75 & 6220 \\
209 & 34.4 & 3.64 & 1.48 & 53.58 & 6119 \\
249 & 34.6 & 3.50 & 1.53 & 71.30 & 6064 \\
296 & 57.5 & 3.56 & 1.51 & 98.45 & 6007 \\
\hline
\end{tabular}

Table 2. List of species considered in the model, along with the internal energies of O excited states (Laher and Gilmore 1990, Kramida et al 2020).

\begin{tabular}{lc}
\hline $\begin{array}{l}\text { Main neutral species } \\
\text { Charged species }\end{array}$ & $\mathrm{CO}_{2}, \mathrm{CO}, \mathrm{O}_{2}, \mathrm{O}\left(2 \mathrm{p}^{43} \mathrm{P}\right), \mathrm{C}$ \\
Metastable states & $\mathrm{e}, \mathrm{O}^{-}, \mathrm{CO}_{2}^{+}, \mathrm{CO}^{+}, \mathrm{O}_{2}^{+}, \mathrm{O}^{+}, \mathrm{C}^{+}$ \\
& $\mathrm{O}\left(2 \mathrm{p}^{41} \mathrm{D}\right)(1.97 \mathrm{eV}), \mathrm{O}\left(2 \mathrm{p}^{41} \mathrm{~S}\right)(4.19 \mathrm{eV})$ \\
Radiative states & $\mathrm{O}\left(3 \mathrm{~s}^{5} \mathrm{~S}^{0}\right)(9.15 \mathrm{eV}), \mathrm{O}\left(3 \mathrm{~s}^{3} \mathrm{~S}^{0}\right)(9.52 \mathrm{eV})$ \\
& $\mathrm{O}\left(3 \mathrm{p}^{5} \mathrm{P}\right)(10.74 \mathrm{eV}), \mathrm{O}\left(3 \mathrm{p}^{3} \mathrm{P}\right)(10.99 \mathrm{eV})$ \\
& $\mathrm{O}\left(3 \mathrm{~d}^{5} \mathrm{D}^{0}\right)(12.08 \mathrm{eV}), \mathrm{O}\left(3 \mathrm{~d}^{3} \mathrm{D}^{0}\right)(12.09 \mathrm{eV})$ \\
\hline
\end{tabular}

and B. Furthermore, that is also the case for $T_{\mathrm{g}, \max }$ and, as such, the degree and extension of dissociation in these plasmas are also in between those of condition A (figure 2) and those of condition B (figure 3).

\section{Numerical model}

\subsection{D-radial plasma fluid model}

Resolving discharge parameters in space requires at least a one-dimensional (1D) description of the $\mathrm{CO}_{2}$ conversion $\mathrm{MW}$ plasma. In this work, we have developed and used an in-house 1D-radial fluid model addressing the plasma at the core axial position, i.e. the position of the centre of the waveguide. The species in table 2 are considered.

The model consists in solving for every species $k$ the steadystate mass balance equations of the form:

$$
\begin{aligned}
\nabla \cdot \boldsymbol{\Gamma}_{\mathbf{k}} & =\frac{1}{r} \frac{\mathrm{d}}{\mathrm{d} r}\left(-r D_{k} \frac{\mathrm{d}}{\mathrm{d} r}\left[y_{k} \rho\right]\right)+\left(\frac{\Gamma_{\text {out }, k}}{L}-\frac{\Gamma_{\text {in }, k}}{L}\right)_{z} \\
& =m_{k}\left(\sum_{j} S_{j, k}-\sum_{i} L_{i, k}\right),
\end{aligned}
$$

where $r$ is the radial coordinate, $y_{k}$ is the mass fraction of species $k$ and $\rho$ is the total mass density of the mixture $\left(\rho=\sum_{k} m_{k} n_{k}=\sum_{k} y_{k} \rho\right) . \Gamma_{k}, D_{k}, n_{k}$ and $m_{k}$ are, respectively, the flux of mass density, the mass diffusion coefficient, the number density and the particle mass of species $k . S_{j, k}$ and $L_{i, k}$ are, respectively, the chemistry source and loss terms associated to reactions $j$ and $i$ acting on species $k$. The reactor under study is characterized by vortex-stabilization, a recirculation zone, turbulent transport and, therefore, complex flow patterns. As such, the local transport is largely unknown and in this model, motivated by the work in Wolf et al (2020a), we describe it as being mostly determined by laminar and turbulent mass diffusion. The model therefore consists mostly of a reaction-diffusion description. An axial convective transport term is included as in Wolf et al (2020a), Viegas et al (2020), in the form of $\left(\frac{\Gamma_{\mathrm{out}, k}}{L}-\frac{\Gamma_{\mathrm{in}, k}}{L}\right)$, where $L$ is the plasma length taken from the emission intensity as $L=L_{777}, \Gamma_{\mathrm{in}, k}$ is the flux of $\mathrm{CO}_{2}$ entering the plasma length, and $\Gamma_{\text {out }, k}$ is the flux of every product exiting the plasma. These fluxes are calculated from the conservation of mass flow rate as:

$$
\begin{aligned}
& \Gamma_{k, z}(r)=m_{k} n_{k}(r) v_{z}(r) \\
& v_{z}(r)=\frac{\dot{m}}{\rho(r) A}
\end{aligned}
$$

where $v_{z}(r)$ is the axial convective velocity at position $r, \dot{m}$ is the input mass flow rate of $\mathrm{CO}_{2}$ at room-temperature and at reactor pressure and $A$ is the tube cross section $A=\pi(13.5$ $\mathrm{mm})^{2}$.

As the electric field associated to the MW propagation in transverse-electric mode in the experiments is not axisymmetric, this model does not include the simulation of electric field through Maxwell's equations. Instead, an electron density profile $n_{\mathrm{e}, \mathrm{IN}}(r)$ (considering equation (2) dependent on $s$ ) is assumed and $E / n_{\mathrm{g}}$ at each position $r$ is self-consistently calculated by iterating this parameter until the electron density $n_{\mathrm{e}}(r)$ matches $n_{\mathrm{e}, \mathrm{IN}}(r) . n_{\mathrm{e}}(r)$ is obtained from the ion densities through a quasi-neutrality assumption. As such, only the electric field magnitude is considered, and not its direction, which leads us to neglecting electric drift and charge separation. The convergence criteria of the simulation are twofold. One criterion consists in matching $n_{\mathrm{e}}(r)$ with $n_{\mathrm{e}, \mathrm{IN}}(r)$ within $1 \%$, for every position where $n_{\mathrm{e}, \mathrm{IN}}(r)>7 \times 10^{16} \mathrm{~m}^{-3}$. We should notice that this tolerance is lower than the experimental uncertainty. For positions with lower electron density, $n_{\mathrm{e}}(r)$ is not required to match $n_{\mathrm{e}, \mathrm{IN}}(r)$ and the reduced electric field $E / n_{\mathrm{g}}$ is assumed to be $1 \mathrm{Td}$, taken as a minimum value in the simulations. The other convergence criterion consists in imposing a solver tolerance for residuals of mass fractions of $10^{-6}$. The model takes quantities from experiments presented in section 2 as input: $p, L_{777}$, flow rate, $n_{\mathrm{e}, \mathrm{IN}}(r)$ (where $n_{\mathrm{e} \Lambda}$ and $\Lambda_{777}$ are included), $T_{\mathrm{g}}(r)$ and radially-resolved composition. Concerning $T_{\mathrm{g}}(r)$ and composition, a linear interpolation is taken in between measured points. For $r>10 \mathrm{~mm}$, where measurements are not available, the values at $r=10 \mathrm{~mm}$ are assumed. The influence of experimental uncertainties associated to quantities used as input in the model, such as $n_{\mathrm{e}}$ and $T_{\mathrm{g}}$, in plasma chemistry simulation results has been put forward in Viegas et al (2020). As the electron density is obtained from quasi-neutrality, the simulations solve equation (11) for a total of 15 species: $\mathrm{C}, \mathrm{O}^{-}, \mathrm{CO}_{2}^{+}, \mathrm{CO}^{+}, \mathrm{O}_{2}^{+}, \mathrm{O}^{+}, \mathrm{C}^{+}$and the metastable and radiative excited states of atomic oxygen. The mass fraction of ground-state $\mathrm{O}\left(2 \mathrm{p}^{4}{ }^{3} \mathrm{P}\right)$ is obtained from subtracting the mass fractions of the $\mathrm{O}$ excited states $\mathrm{O}(i)$ to the input one of $\mathrm{O}$ as $y_{\mathrm{O}\left(2 \mathrm{p}^{43} \mathrm{P}\right)}=y_{\mathrm{O}}-\sum_{i} y_{\mathrm{O}(i)}$. The measured molar fractions of $\mathrm{CO}_{2}, \mathrm{CO}, \mathrm{O}_{2}$ and $\mathrm{O}$ are given as input to the model and allow to calculate $\rho(r)$ according to the ideal 
gas law. Then, the mass fractions of these species are slightly adjusted during the simulations in order to preserve the ideal gas law. The reactions corresponding to the chemistry source terms $S_{k, j}$ are described in section 3.2.

As radial transport in the reactor under study is largely unknown, we describe it for the 15 species in the same way as in Wolf et al (2020a), where simulation results have found good experimental agreement. Radial transport is described in this model through laminar and turbulent Fickian mass diffusion, with the diffusion coefficient of each species $k$ defined as the sum of a laminar coefficient $D_{\mathrm{L}, k}$ and an effective turbulent coefficient $D_{\mathrm{T}}$ :

$$
D_{k}(r)=D_{\mathrm{L}, k}(r)+D_{\mathrm{T}}(r) .
$$

The turbulent diffusion coefficient is taken from Wolf et al (2020a), where it is defined independently of the mixture as:

$$
D_{\mathrm{T}}(r)=\frac{\nu_{\mathrm{T}}(r)}{\mathrm{Sc}_{\mathrm{T}}}
$$

with constant turbulent $\mathrm{Schmidt}$ number $\mathrm{Sc}_{\mathrm{T}}=0.71$ (Yimer et al 2002), and turbulent viscosity $\nu_{\mathrm{T}}(r)$. The same formulation has been adopted in Synek et al (2015). In Wolf et al (2020a), in agreement with computational fluid dynamics simulations of the vortex flow including a plasma heat source, it is stated that the radial variation in $\nu_{\mathrm{T}}(r)$ can be approximated by a quadratic function of $r$ :

$$
\nu_{\mathrm{T}}(r)=\nu_{\mathrm{T} \text {,peak }} \cdot\left(1-\frac{r^{2}}{R_{\text {tube }}^{2}}\right)
$$

Then, the value on the axis $\nu_{\mathrm{T}, \text { peak }}$ has been found in Wolf et al (2020a) for several conditions of pressure and flow and for an input power of $1 \mathrm{~kW}$, as the value that best allows to retrieve the experimental peak of $T_{\mathrm{g}}$, obtained from Doppler broadening measurements of the $777 \mathrm{~nm}$ atomic oxygen line emission (Wolf et al 2019). An improvement is made with respect to the case in Wolf et al (2020a), as the laminar diffusion coefficient in this work is obtained for each species $k$ through a mixture-averaged multicomponent approach, as in Hirschfelder and Curtiss (1949), Giovangigli (1990), Synek et al (2015):

$$
D_{\mathrm{L}, k}(r)=\frac{1-y_{k}}{\sum_{l \neq k} \frac{x_{1}}{\mathcal{D}_{k, l}}},
$$

where $x_{l}$ is the molar fraction of species $l$ and $\mathcal{D}_{k, l}$ is the binary diffusion coefficient of the $(l, k)$ pair of species. The binary diffusion coefficients have been computed for all neutral-neutral, ion-neutral and electron-neutral interactions from Chapman-Enskog theory (Capitelli et al 2013), using the collision integrals in Laricchiuta et al (2009). In the conditions of this work, in the plasma core region, for ions and neutrals, $D_{\mathrm{L}, k}$ and $D_{\mathrm{T}}$ are of the same order of magnitude, around $10^{-2} \mathrm{~m}^{2} \mathrm{~s}^{-1}$.

The MW absorbed power density $P_{\mathrm{abs}}(r)$ is calculated as an output through the steady-state electron energy balance equation (Alves et al 2018):

$$
\begin{aligned}
P_{\mathrm{abs}}(r)= & \left(\frac{P_{\mathrm{el}}}{n_{\mathrm{g}}}(r)+\frac{P_{\text {inel }}}{n_{\mathrm{g}}}(r)+\frac{P_{\text {growth }}}{n_{\mathrm{g}}}(r)\right) n_{\mathrm{g}}(r) n_{\mathrm{e}}(r) \\
& +\frac{n_{\mathrm{e}}(r) \epsilon(r) v_{\mathrm{z}}(r)}{L} \\
& +\frac{\mathrm{d}}{\mathrm{d} r}\left(-\frac{5}{3} D_{\mathrm{e}}(r) \frac{\mathrm{d}}{\mathrm{d} r}\left[n_{\mathrm{e}}(r) \epsilon(r)\right]\right),
\end{aligned}
$$

where $P_{\text {el }}, P_{\text {inel }}$ and $P_{\text {growth }}$ are the components of power lost by electrons through elastic, conservative inelastic and nonconservative inelastic collisions, respectively, calculated from the solver for electron kinetics and dependent on $E / n_{\mathrm{g}}$ and gas mixture. $\epsilon$ is the mean electron energy also calculated from the electron kinetics solver and $D_{\mathrm{e}}$ is the electron diffusion coefficient, calculated from equations (14)-(17). We should notice that the formulation used for the diffusive term in equation (18) is an approximation based on the assumption of a Maxwellian electron velocity distribution function and an energy-independent momentum-transfer collision frequency (Alves et al 2018). However, in the studied conditions, the transport terms (mostly diffusive) in equation (18) have a weight of the order of only $2 \%$ on the calculation of $P_{\text {abs }}$, that is mostly defined by the collisional electron losses. Hence, formulating the transport terms differently would have a negligible effect on $P_{\text {abs }}(r)$.

The electron kinetics solver is the Monte Carlo flux (MCF) code developed and described in Vialetto et al (2019, 2020), based on the method introduced in Schaefer and Hui (1990). It is used in this model to calculate the power loss terms and the electron mean energy in equation (18) and the electronimpact rate coefficients that take part in the source term calculations. The MCF code can simulate electrons in any arbitrary gas mixture, including any population of excited states for atoms/molecules. Moreover, it is consistent with the cross sections set for electron impact used in this work. The importance of these considerations for the calculation of rate coefficients has been demonstrated in Viegas et al (2020), Vialetto et al (2020). The MCF solution is coupled to the 1D-radial model in a similar way as it was coupled to the zero-dimensional model in Viegas et al (2020). In 11 equally-spaced positions in the domain, the MCF solution is obtained for 21 values of reduced electric field linearly spaced between 1 and $200 \mathrm{Td}$. Then, the solution is interpolated in space. As such, the calculated electron parameters are functions of the local $E / n_{\mathrm{g}}$ and of position. The electron kinetics calculations take as input parameters the gas pressure and the frequency of the field, as well as the local gas temperature and composition, consistently with the plasma model and the experimental conditions reported in section 2 . The vibrational states of molecules are taken into account in electron collisions by assuming that they are populated according to a Boltzmann distribution at $T_{\mathrm{g}}$, as in Viegas et al (2020). The importance of considering these populations for electron kinetics in $\mathrm{CO}_{2}$ has been demonstrated for example in Grofulović et al (2016), Vialetto et al (2020), Silva et al (2020). The MCF solutions are first obtained for the input molar fractions of the main species $\mathrm{CO}_{2}, \mathrm{CO}, \mathrm{O}_{2}$ and $\mathrm{O}$. Then, after the plasma simulation converges, the $\mathrm{MCF}$ solutions are updated and the plasma simulation is run again. 
Table 3. List of atomic oxygen reactions: electron-impact excitation, deexcitation, ionisation and dissociation; electron-ion recombination; and radiative emission taking self-absorption into account. $\mathrm{O}(i)$ and $\mathrm{O}(j)$ represent any state of atomic oxygen listed in table 2, except when a note is added. The units of rate coefficients for radiative emission are $\mathrm{s}^{-1}$, and for two-body reactions are $\mathrm{cm}^{3} \mathrm{~s}^{-1} . E / n_{\mathrm{g}}$ is the reduced electric field; $r$ is the radial position, corresponding to a local gas mixture and temperature; and $T_{\mathrm{e}}$ is the electron temperature in $\mathrm{eV}^{\mathrm{a}}{ }^{\mathrm{a}} \mathrm{b}$

\begin{tabular}{lccc}
\hline $\mathrm{Nbr}$ & Reaction & Rate coefficient & References \\
\hline $\mathrm{O} 1$ & $\mathrm{e}+\mathrm{O}(i) \leftrightarrow \mathrm{e}+\mathrm{O}(j)$ & $k\left(E / n_{\mathrm{g}}, r\right)$ & {$[1,2,3]$} \\
$\mathrm{O} 2$ & $\mathrm{e}+\mathrm{O}(i) \rightarrow \mathrm{e}+\mathrm{O}^{+}$ & $k\left(E / n_{\mathrm{g}}, r\right)$ & {$[1]$} \\
$\mathrm{O} 3$ & $\mathrm{e}+\mathrm{CO}_{2} \rightarrow \mathrm{e}+\mathrm{CO}+\mathrm{O}\left(2 \mathrm{p}^{4}{ }^{1} \mathrm{~S}\right)$ & $k\left(E / n_{\mathrm{g}}, r\right)$ & {$[4]$} \\
$\mathrm{O} 4$ & $\mathrm{e}+\mathrm{CO} \rightarrow \mathrm{e}+\mathrm{C}+\mathrm{O}\left(2 \mathrm{p}^{4}{ }^{1} \mathrm{~S}\right)$ & $k\left(E / n_{\mathrm{g}}, r\right)$ & {$[4]$} \\
$\mathrm{O} 5$ & $\mathrm{e}+\mathrm{O}_{2} \rightarrow \mathrm{e}+\mathrm{O}\left(2 \mathrm{p}^{4}{ }^{3} \mathrm{P}\right)+\mathrm{O}(i)$ & {$[2,4-8]$} \\
$\mathrm{O} 6$ & $\mathrm{e}+\mathrm{O}_{2}^{+} \rightarrow \mathrm{O}\left(2 \mathrm{p}^{4}{ }^{3} \mathrm{P}\right)+\mathrm{O}\left(2 \mathrm{p}^{4}{ }^{3} \mathrm{P}\right)$ & $5.17 \times 10^{-9} \times T_{\mathrm{e}}^{-1}$ & {$[9,10]$} \\
$\mathrm{O} 7$ & $\mathrm{e}+\mathrm{O}_{2}^{+} \rightarrow \mathrm{O}\left(2 \mathrm{p}^{4}{ }^{3} \mathrm{P}\right)+\mathrm{O}\left(2 \mathrm{p}^{4}{ }^{1} \mathrm{D}\right)$ & $1.51 \times 10^{-8} \times T_{\mathrm{e}}^{-0.7}$ & {$[9,11]$} \\
$\mathrm{R} 1$ & $\mathrm{O}\left(3 \mathrm{p}{ }^{5} \mathrm{P}\right) \rightarrow \mathrm{O}\left(3 \mathrm{~s}^{5} \mathrm{~S}^{0}\right)(777 \mathrm{~nm})$ & $\tau_{\text {rad1 }}^{-1}$ & {$[12-14]$} \\
$\mathrm{R} 2$ & $\mathrm{O}\left(3 \mathrm{p}^{3} \mathrm{P}\right) \rightarrow \mathrm{O}\left(3 \mathrm{~s}^{3} \mathrm{~S}^{0}\right)(845 \mathrm{~nm})$ & $\tau_{\text {rad2 }}^{-1}$ & {$[12-14]$} \\
$\mathrm{R} 3$ & $\mathrm{O}\left(3 \mathrm{~d}^{5} \mathrm{D}^{0}\right) \rightarrow \mathrm{O}\left(3 \mathrm{p}^{5} \mathrm{P}\right)(926 \mathrm{~nm})$ & $\tau_{\text {rad3 }}^{-1}$ & {$[12-14]$} \\
$\mathrm{R} 4$ & $\mathrm{O}\left(3 \mathrm{~d}^{3} \mathrm{D}^{0}\right) \rightarrow \mathrm{O}\left(3 \mathrm{p}^{3} \mathrm{P}\right)(1128 \mathrm{~nm})$ & $\tau_{\text {rad4 }}^{-1}$ & {$[12-14]$} \\
R5 & $\mathrm{O}\left(3 \mathrm{~d}^{3} \mathrm{D}^{0}\right) \rightarrow \mathrm{O}\left(2 \mathrm{p}^{4}{ }^{3} \mathrm{P}\right)(102 \mathrm{~nm})$ & $\tau_{\text {rad5 }}^{-1}$ & {$[12-14]$} \\
R6 & $\mathrm{O}\left(3 \mathrm{~s}^{3} \mathrm{~S}^{0}\right) \rightarrow \mathrm{O}\left(2 \mathrm{p}^{4}{ }^{3} \mathrm{P}\right)(130 \mathrm{~nm})$ & $\tau_{\text {rad6 }}^{-1}$ & {$[12-14]$} \\
\hline
\end{tabular}

aReferences: [1] = Laher and Gilmore (1990); [2] = Alves et al (2016); [3] = Barklem (2007); [4] =

McConkey et al (2008); [5] = Erdman and Zipf (1987); [6] = Lawrence (1970); [7] = Itikawa (2009); [8] = Kanik et al (2003); [9] = Annušová et al (2018); [10] = Kossyi et al (1992); [11] = Eliasson and Kogelschatz (1986); [12] = NIST database, Kramida et al (2020); [13] = Fiebrandt et al (2020); [14] = Mewe (1967).

${ }^{b}$ Notes on the states considered: $\mathrm{a}-\mathrm{O}\left(2 \mathrm{p}^{4}{ }^{1} \mathrm{D}\right), \mathrm{O}\left(2 \mathrm{p}^{4}{ }^{1} \mathrm{~S}\right), \mathrm{O}\left(3 \mathrm{~s}^{5} \mathrm{~S}^{0}\right), \mathrm{O}\left(3 \mathrm{~s}^{3} \mathrm{~S}^{0}\right), \mathrm{O}\left(3 \mathrm{p}{ }^{5} \mathrm{P}\right)$ and $\mathrm{O}\left(3 \mathrm{p}^{3} \mathrm{P}\right)$.

The update takes into account the calculated densities of $\mathrm{C}$ and of the atomic oxygen excited states, that have influence on the EEDF through both superelastic and stepwise inelastic collisions. As the densities of these species are much lower than those of the main species (whose fractions are fixed) and have only a minor effect on the EEDF, the MCF solutions are updated only once. In this way, electron kinetics is coupled self-consistently with plasma simulations.

The equations in this model are solved with a finite volume discretisation in a uniform grid, where diffusive mass fluxes are discretised using a central difference scheme. These are solved using the tridiagonal matrix algorithm for matrix inversion. Homogeneous Neumann boundary conditions for the radial diffusive fluxes are taken at $r=0$ and at the tube wall. In this work the $13.5 \mathrm{~mm}$ radial domain is divided into 41 cells of $0.33 \mathrm{~mm}$ size each. The numerical solution is obtained in a single central processing unit with a calculation time ranging typically between 1 and 7 days. One simulation has been performed with 81 cells of $0.17 \mathrm{~mm}$ size each and has produced a relative difference in results up to $5 \%$ and an increase in calculation time of a factor 5.5, with respect to the same case with 41 cells. The calculation time is mostly due to the MCF calculations taking approximately $11 \mathrm{~h}$ ( $1 \mathrm{~h}$ per position) and to the very high number of iterations required to fulfil the convergence criteria, which is usually between 2 million and 20 million. On the one hand, the high number of iterations is justified by the use of a small under-relaxation factor of $10^{-5}$, necessary for the resolution of the very stiff non-linear system of equations imposed by the complex chemistry in plasmas (Patankar 1980). On the other hand, we should notice that it is very computationally expensive to simultaneously match $n_{\mathrm{e}}$ with $n_{\mathrm{e}, \mathrm{IN}}$ in the whole domain, but it is of fundamental impor- tance for the current study of spatial resolution of discharge parameters.

\section{2. $\mathrm{O}$ atom collisional-radiative model in $\mathrm{CO}_{2}$ conversion plasma}

In this section, the reaction scheme used in the model is presented. The reactions considered in Viegas et al (2020) are adopted in this work. These include reactions for neutral thermal chemistry based on the GRI-MECH 3.0 database (Smith et al 2018) and for charged particle kinetics based on the reaction schemes of Koelman et al (2017), Kozák and Bogaerts (2014). Charged particle kinetics includes electron-impact ionisation, attachment and dissociation, electron-ion recombination, ion transfers, detachment, ion-ion recombination and associative ionisation. The rate coefficients and electronimpact cross sections considered for those reactions are adopted from Viegas et al (2020), Vialetto et al (2020). Electron-electron collisions are not considered, as they are not expected to affect rate coefficients in the conditions of interest beyond an increase of 5\%. As in Viegas et al (2020), molecules are vibrationally populated according to a Boltzmann distribution at $T_{\mathrm{g}}$ for which concerns electron kinetics, in agreement with the temperature measurements in van den Bekerom et al (2020), van de Steeg et al (2020). Nevertheless, their excited states, as those of $\mathrm{C}$, are not considered for chemical calculations. We thus assume that the radiative emission of $\mathrm{O}$ in this plasma is independent of those states and is mostly due to processes affecting exclusively $\mathrm{O}$ states. As such, a CRM for atomic oxygen and the excited states listed in table 2 is added in this work, inspired on the recent works of Caplinger and Perram (2020) and of Fiebrandt et al (2020). As in Viegas et al (2020), no wall reactions are considered, since the contracted 
Table 4. List of atomic oxygen quenching reactions. $\mathrm{O}(i)$ and $\mathrm{O}(j)$ represent any state of atomic oxygen listed in table 2 and $M$ represents any neutral species, except when a note is added. The units of rate coefficients are $\mathrm{cm}^{3} \mathrm{~s}^{-1} . T_{\mathrm{g}}$ is the gas temperature in $\mathrm{K} .{ }^{\mathrm{a}, \mathrm{b}}$

\begin{tabular}{|c|c|c|c|}
\hline Nbr & Reaction & Rate coefficient & References \\
\hline Q1 & $\mathrm{O}\left(3 \mathrm{p}^{3} \mathrm{P}\right)+\mathrm{M} \rightarrow \mathrm{O}\left(3 \mathrm{p}{ }^{5} \mathrm{P}\right)+\mathrm{M}$ & $6 \times 10^{-11}$ & {$[15,16]$} \\
\hline Q2 & $\mathrm{O}\left(3 \mathrm{~d}^{3} \mathrm{D}^{0}\right)+\mathrm{M} \rightarrow \mathrm{O}\left(3 \mathrm{~d}^{5} \mathrm{D}^{0}\right)+\mathrm{M}$ & $6 \times 10^{-11}$ & {$[15,16]$} \\
\hline Q3 & $\mathrm{O}\left(2 \mathrm{p}^{4}{ }^{1} \mathrm{D}\right)+\mathrm{M}^{\mathrm{b}} \rightarrow \mathrm{O}\left(2 \mathrm{p}^{4}{ }^{3} \mathrm{P}\right)+\mathrm{M}^{\mathrm{b}}$ & $2.60 \times 10^{-11} \times \exp ^{67 / T_{\mathrm{g}}}+10^{-12}$ & {$[15,17]$} \\
\hline Q4 & $\mathrm{O}\left(2 \mathrm{p}^{4}{ }^{1} \mathrm{D}\right)+\mathrm{O} \rightarrow \mathrm{O}\left(2 \mathrm{p}^{4}{ }^{3} \mathrm{P}\right)+\mathrm{O}$ & $8 \times 10^{-12}$ & {$[15,17]$} \\
\hline Q5 & $\mathrm{O}\left(2 \mathrm{p}^{4}{ }^{1} \mathrm{D}\right)+\mathrm{C} \rightarrow \mathrm{O}\left(2 \mathrm{p}^{4}{ }^{3} \mathrm{P}\right)+\mathrm{C}$ & $2.30 \times 10^{-11}$ & {$[15,17]$} \\
\hline Q6 & $\mathrm{O}\left(2 \mathrm{p}^{4}{ }^{1} \mathrm{~S}\right)+\mathrm{M}^{\mathrm{b}} \rightarrow \mathrm{O}\left(2 \mathrm{p}^{4}{ }^{3} \mathrm{P}\right)+\mathrm{M}^{\mathrm{b}}$ & $4 \times 10^{-12} \times \exp \left(-865 / T_{\mathrm{g}}\right)$ & {$[15,17]$} \\
\hline Q7 & $\mathrm{O}\left(2 \mathrm{p}^{4{ }^{1}} \mathrm{~S}\right)+\mathrm{O} \rightarrow \mathrm{O}\left(2 \mathrm{p}^{4}{ }^{1} \mathrm{D}\right)+\mathrm{O}\left(2 \mathrm{p}^{4}{ }^{1} \mathrm{D}\right)$ & $5 \times 10^{-11} \times \exp \left(-301 / T_{\mathrm{g}}\right)$ & {$[15,17]$} \\
\hline Q8 & $\mathrm{O}\left(2 \mathrm{p}^{4}{ }^{1} \mathrm{~S}\right)+\mathrm{C} \rightarrow \mathrm{O}\left(2 \mathrm{p}^{4}{ }^{3} \mathrm{P}\right)+\mathrm{C}$ & $10^{-12}$ & {$[15,17]$} \\
\hline Q9 & $\mathrm{O}(i)^{\mathrm{c}}+\mathrm{O}_{2} \rightarrow \mathrm{O}\left(2 \mathrm{p}^{4}{ }^{3} \mathrm{P}\right)+\mathrm{O}_{2}$ & $2.20 \times 10^{-10}$ & {$[15,18]$} \\
\hline Q10 & $\mathrm{O}(i)^{\mathrm{c}}+\mathrm{CO}_{2} \rightarrow \mathrm{O}\left(2 \mathrm{p}^{4}{ }^{3} \mathrm{P}\right)+\mathrm{CO}_{2}$ & $5.30 \times 10^{-10}$ & {$[15,18]$} \\
\hline Q11 & $\mathrm{O}(i)^{\mathrm{c}}+\mathrm{CO} \rightarrow \mathrm{O}\left(2 \mathrm{p}^{4}{ }^{3} \mathrm{P}\right)+\mathrm{CO}$ & $8.30 \times 10^{-11}$ & {$[15,18]$} \\
\hline Q12 & $\mathrm{O}(i)^{\mathrm{c}}+\mathrm{O} \rightarrow \mathrm{O}\left(2 \mathrm{p}^{4}{ }^{3} \mathrm{P}\right)+\mathrm{O}$ & $2.20 \times 10^{-10}$ & {$[15,18]$} \\
\hline Q13 & $\mathrm{O}(i)^{\mathrm{c}}+\mathrm{C} \rightarrow \mathrm{O}\left(2 \mathrm{p}^{4}{ }^{3} \mathrm{P}\right)+\mathrm{C}$ & $3.50 \times 10^{-11}$ & {$[15,18]$} \\
\hline Q14 & $\mathrm{O}(i)^{\mathrm{d}}+\mathrm{M}^{\mathrm{e}} \rightarrow \mathrm{O}\left(2 \mathrm{p}^{4}{ }^{3} \mathrm{P}\right)+\mathrm{M}^{\mathrm{e}}$ & $10.80 \times 10^{-10}$ & {$[15,16]$} \\
\hline Q15 & $\mathrm{O}(i)^{\mathrm{f}}+\mathrm{M}^{\mathrm{e}} \rightarrow \mathrm{O}\left(2 \mathrm{p}^{4}{ }^{3} \mathrm{P}\right)+\mathrm{M}^{\mathrm{e}}$ & $9.30 \times 10^{-10}$ & {$[15,19]$} \\
\hline Q16 & $\mathrm{O}(i)^{\mathrm{g}}+\mathrm{C} \rightarrow \mathrm{O}\left(2 \mathrm{p}^{4}{ }^{3} \mathrm{P}\right)+\mathrm{C}$ & $5.90 \times 10^{-10}$ & {$[15,19]$} \\
\hline
\end{tabular}

a References: [15] = Caplinger and Perram (2020); [16] = Dagdigian et al (1988); [17] = Gordiets et al (1995); [18] = Mori et al (1992); [19] = Niemi et al (2001).

${ }^{b}$ Notes on the species or states considered: $\mathrm{b}-\mathrm{CO}_{2}, \mathrm{CO}$ and $\mathrm{O}_{2} ; \mathrm{c}-\mathrm{O}\left(3 \mathrm{~s}^{5} \mathrm{~S}^{0}\right)$ and $\mathrm{O}\left(3 \mathrm{~s}^{3} \mathrm{~S}^{0}\right) ; \mathrm{d}-\mathrm{O}\left(3 \mathrm{p}{ }^{5} \mathrm{P}\right)$ and $\mathrm{O}\left(3 \mathrm{~d}^{5} \mathrm{D}^{0}\right) ; \mathrm{e}-\mathrm{CO}_{2}, \mathrm{CO}, \mathrm{O}_{2}$ and $\mathrm{O} ; \mathrm{f}-\mathrm{O}\left(3 \mathrm{p}^{3} \mathrm{P}\right)$ and $\mathrm{O}\left(3 \mathrm{~d}^{3} \mathrm{D}^{0}\right) ; \mathrm{g}-\mathrm{O}\left(3 \mathrm{p}^{5} \mathrm{P}\right), \mathrm{O}\left(3 \mathrm{p}^{3} \mathrm{P}\right), \mathrm{O}\left(3 \mathrm{~d}^{5} \mathrm{D}^{0}\right)$ and $\mathrm{O}(3 \mathrm{~d}$ $\left.{ }^{3} \mathrm{D}^{0}\right)$.

MW plasma has no direct interaction with the reactor walls. Together with the reaction scheme in Viegas et al (2020), a total of 225 reactions is considered in our model. The reactions of the CRM are listed in tables 3 and 4 and have been defined according to the following considerations:

- The cross sections for excitation and ionisation of groundstate atomic oxygen by electron impact have been retrieved from Laher and Gilmore (1990), where a critical review of experimental and theoretical work on this subject has been presented. Superelastic cross sections are calculated with the formula of Klein-Rosseland (Capitelli et al 2015), by considering micro-reversibility. The electron kinetics solver considers separate transitions between the ground-state and all the non-Rydberg states and all the Rydberg states with a $\mathrm{O}^{+}\left({ }^{4} \mathrm{~S}^{0}\right)$ core, which is consistent with the set in Alves et al (2016) where all these Rydberg states are lumped together. Rydberg states with $\mathrm{O}^{+}\left({ }^{2} \mathrm{D}^{0}\right)$ and $\mathrm{O}^{+}\left({ }^{2} \mathrm{P}^{0}\right)$ cores are taken into account as lumped states, as in Alves et al (2016). The double ionisation cross section presented in Laher and Gilmore (1990) is not considered, due to its very high threshold of around $50 \mathrm{eV}$.

- The CRM considers that $\mathrm{O}\left(3 \mathrm{~d}^{5} \mathrm{D}^{0}\right)$ electron-impact excitation includes the excitation to $\mathrm{O}\left(4 \mathrm{~s}^{5} \mathrm{~S}^{0}\right), \mathrm{O}\left(4 \mathrm{p}{ }^{5} \mathrm{P}\right)$ and $\mathrm{O}\left(4 \mathrm{~d}^{5} \mathrm{D}^{0}\right)$, as a way to account for radiative cascading of all quintuplet states above $\mathrm{O}\left(3 \mathrm{p}{ }^{5} \mathrm{P}\right)$, as in Fiebrandt et al (2020). This approach assumes that the upper states relax via radiative processes. The same is done for $\mathrm{O}(3 \mathrm{~d}$ $\left.{ }^{3} \mathrm{D}^{0}\right)$, including states $\mathrm{O}\left(4 \mathrm{~s}^{3} \mathrm{~S}^{0}\right), \mathrm{O}\left(4 \mathrm{p}^{3} \mathrm{P}\right)$ and $\mathrm{O}\left(4 \mathrm{~d}^{3} \mathrm{D}^{0}\right)$. The relevance of cascade emission has been put forward by both Fiebrandt et al (2020) and Caplinger and Perram (2020).
- Electron-impact collisions with excited states, shown to be relevant in Caplinger and Perram (2020), are also considered. The elastic cross sections are considered as being the same as for the ground-state, taken from Alves et al (2016). The following stepwise excitation collision cross sections have been retrieved from Barklem (2007), as in Caplinger and Perram (2020), Fiebrandt et al (2020): $\mathrm{O}\left(2 \mathrm{p}^{4}{ }^{1} \mathrm{D}\right) \rightarrow \mathrm{O}\left(2 \mathrm{p}^{4}{ }^{1} \mathrm{~S}\right), \mathrm{O}\left(3 \mathrm{~s}^{3} \mathrm{~S}^{0}\right), \mathrm{O}\left(3 \mathrm{p}{ }^{3} \mathrm{P}\right) ; \mathrm{O}\left(2 \mathrm{p}^{4}{ }^{1} \mathrm{~S}\right)$ $\rightarrow \mathrm{O}\left(3 \mathrm{p}^{3} \mathrm{P}\right) ; \mathrm{O}\left(3 \mathrm{~s}^{5} \mathrm{~S}^{0}\right) \rightarrow \mathrm{O}\left(3 \mathrm{~s}^{3} \mathrm{~S}^{0}\right), \mathrm{O}\left(3 \mathrm{p}^{5} \mathrm{P}\right), \mathrm{O}\left(3 \mathrm{p}{ }^{3} \mathrm{P}\right)$; $\mathrm{O}\left(3 \mathrm{~s}^{3} \mathrm{~S}^{0}\right) \rightarrow \mathrm{O}\left(3 \mathrm{p}{ }^{5} \mathrm{P}\right), \mathrm{O}\left(3 \mathrm{p}{ }^{3} \mathrm{P}\right) ; \mathrm{O}\left(3 \mathrm{p}{ }^{5} \mathrm{P}\right) \rightarrow \mathrm{O}\left(3 \mathrm{p}{ }^{3} \mathrm{P}\right)$. They have been calculated in Barklem (2007) through a 38-state R-matrix calculation. In the cases where literature sources did not include data tables, cross sections have been digitised from figures. The remaining stepwise excitation and ionisation cross sections are taken as the same as those for electron impact with the ground-state of O from Laher and Gilmore (1990). The same approach to cascade emission is used for stepwise excitation as for ground-state excitation.

- Electron-impact dissociation of $\mathrm{CO}_{2}$ and $\mathrm{CO}$ (reactions O3-O4), using the cross sections in the review of McConkey et al (2008), produce the $\mathrm{O}\left(2 \mathrm{p}^{4}{ }^{1} \mathrm{~S}\right)$ state.

- The electron-impact cross sections for dissociation of $\mathrm{O}_{2}$ into $\mathrm{O}\left(2 \mathrm{p}^{4}{ }^{3} \mathrm{P}\right)+\mathrm{O}\left(2 \mathrm{p}^{4}{ }^{3} \mathrm{P}\right)$ and $\mathrm{O}\left(2 \mathrm{p}^{4}{ }^{3} \mathrm{P}\right)+\mathrm{O}\left(2 \mathrm{p}^{4}{ }^{1} \mathrm{D}\right)$ (reaction O5), which are the most populated states of atomic oxygen, are taken from Alves et al (2016). Then, for the production of $\mathrm{O}\left(2 \mathrm{p}^{4}{ }^{1} \mathrm{~S}\right), \mathrm{O}\left(3 \mathrm{p}{ }^{5} \mathrm{P}\right)$ and $\mathrm{O}(3 \mathrm{p}$ $\left.{ }^{3} \mathrm{P}\right)(\mathrm{O} 5)$, the dissociative cross sections in McConkey et al (2008) are considered. Finally, we take into account electron-impact dissociation of $\mathrm{O}_{2}$ into the metastables $\mathrm{O}\left(3 \mathrm{~s}^{5} \mathrm{~S}^{0}\right)$ and $\mathrm{O}\left(3 \mathrm{~s}^{3} \mathrm{~S}^{0}\right)$ using the emission cross sections from Itikawa (2009), Kanik et al (2003). This choice of cross sections is the same as in Fiebrandt et al (2020) and 
in Stancu et al (2016) and is similar to the one in Caplinger and Perram (2020). While in Fiebrandt et al (2020) a Maxwellian EEDF is assumed to obtain expressions for rate coefficients, in this model we calculate all electronimpact rate coefficients from the EEDF calculated through the MCF method.

- No kinetic data has been found in literature for production of excited states of $\mathrm{O}$ from dissociative electron-ion recombination. The exceptions are the reactions listed in Annušová et al (2018) and included in table 3 as $\mathrm{O} 6-\mathrm{O} 7$ for $\mathrm{O}_{2}^{+}$recombination. We should notice that this is the dominant ion in the studied plasma (Viegas et al 2020).

- The Einstein coefficients for spontaneous emission are taken from the NIST database (Kramida et al 2020). Only transitions with Einstein coefficient above $10^{5} \mathrm{~s}^{-1}$ have been selected (R1-R6 in table 3 ). The remaining transitions in the NIST database have Einstein coefficient below $10^{4} \mathrm{~s}^{-1}$ and thus have been neglected. In fact, the emission intensities of the transitions with wavelengths 777 $\mathrm{nm}, 845 \mathrm{~nm}$ and $926 \mathrm{~nm}(\mathrm{R} 1-\mathrm{R} 3)$ are measured in the experiments in the $\mathrm{CO}_{2} \mathrm{MW}$ plasma reactor. The measurements are not sensitive to the wavelengths of reactions R4-R6, but the importance of these transitions cannot be excluded.

- The influence of self-absorption on transitions R1-R6 is considered as in Fiebrandt et al (2020), where it has been shown to be important. Radiation emitted from a higher level $p$ to a lower level $k$, of multiplet component $l$, can be reabsorbed by the lower state to repopulate the higher state. This process limits the number of photons effectively travelling through the plasma. In this work we take it into account by adding a local correction called escape factor $\gamma_{p k, l}(r)$, depending on the number density of the lower level at each position $r$, such that every coefficient $\tau_{\text {rad } p k}^{-1}(r)$ in table 3 is defined as:

$$
\tau_{\operatorname{rad} p k}^{-1}(r)=\sum_{l} A_{p k, l} \gamma_{p k, l}(r) \frac{g_{p, l}}{\sum_{l} g_{p, l}},
$$

where $A_{p k, l}$ is the Einstein coefficient of each transition from state $p$ to state $k$, of multiplet component $l$, found in NIST; $\gamma_{p k, l}$ is the associated escape factor and $g_{p, l}$ is the statistical weight of the upper state in the corresponding transition. To calculate $\gamma_{p k, l}$, we use the same approach as in Fiebrandt et al (2020), an approximated empirical formula determined by Mewe (Mewe 1967). In Mewe (1967), the formula assumes homogeneous density profiles of both the upper and the lower state. Then, in Sushkov et al (2013), it has been shown that the approximation is valid with any spatial profile of the states densities, as long as the upper and lower states have similar spatial profiles. It has also been estimated that in the worst case scenario, this empirical formula is approximately a factor of 2-3 off to the correct escape factor. In this work, this method is used due to its simplicity, and, as we use this approximation in the 1D model, we take the local densities at each position $r$, such that:

$$
\begin{aligned}
\gamma_{p k, l}(r) & =\frac{2-\exp \left(-10^{-3} k_{p k, l}(r) \Lambda_{777}\right)}{1+k_{p k, l}(r) \Lambda_{777}} \\
k_{p k, l}(r) & =\frac{\lambda_{p k, l}^{2}}{8 \pi} P_{p k, l}(r) \frac{g_{p, l}}{g_{k, l}} n_{k, l}(r) A_{p k, l} \\
n_{k, l}(r) & =n_{k}(r) \frac{g_{k, l}}{\sum_{l} g_{k, l}} \\
P_{p k, l}(r) & =\lambda_{p k, l} \sqrt{\frac{m_{\mathrm{O}}}{2 \pi k_{\mathrm{B}} T_{\mathrm{g}}(r)}}
\end{aligned}
$$

$k_{p k, l}, \lambda_{p k, l}$ and $P_{p k, l}$ are the absorption coefficient, the wavelength and the spectral line profile of the associated transition. $P_{p k, l}$ has been taken as in Fiebrandt et al (2017), assuming Doppler broadening as the dominant line broadening mechanism. $\Lambda_{777}$ is the measured FWHM of the $777 \mathrm{~nm}$ emission, here taken as an approximate thickness of the plasma in the radial direction, over which photons are absorbed. $m_{\mathrm{O}}$ is the mass of atomic oxygen, $k_{\mathrm{B}}$ is the Boltzmann constant and $n_{k, l}$ is the number density of species $k$ in the multiplet component $l$, estimated from the statistical weights $g_{k, l}$.

- Numerical simulations in the conditions studied in this work show that, using the described approach, the effective escape factors can go down to 0.5 in the case of transitions $\mathrm{R} 1-\mathrm{R} 2$ in the centre of the discharge. The escape factors increase radially to approximately 1 on the edges. For transitions R3-R4, with radiative lower state, the escape factor is always close to 1 . For the cases of transitions R5-R6, where the lower state is the ground-state, the emission is decreased by about $90 \%$ (escape factor of $\sim 0.1)$. The approach used in Santos et al (2014) has also been tested, yielding no self-absorption for R1-R4 and complete self-absorption for R5-R6. It has been discarded, since it is only valid for high levels of radiation trapping.

- For the quenching rate coefficients, we use those compiled in Caplinger and Perram (2020). We should notice that many rate coefficients are not reported in literature and many are measured at $300 \mathrm{~K}$ only and thus have no temperature dependence. The quenching rate coefficient for excitation transfer Q1 (table 4) is taken from Dagdigian et al (1988), where it has been measured with $\mathrm{O}_{2}$ as collision partner from the ratio of the $777 \mathrm{~nm}$ and 845 $\mathrm{nm}$ emission intensities. The important role of this reaction has been put forward in Caplinger and Perram (2020). As this rate coefficient hasn't been found in literature for the other collision partners $\left(\mathrm{CO}_{2}, \mathrm{CO}, \mathrm{O}\right.$ and $\left.\mathrm{C}\right)$, we have taken the same rate coefficient for all collision partners. In Morillo-Candas et al (2019), the quenching rate coefficient of reaction $\mathrm{O}\left(3 \mathrm{p}{ }^{5} \mathrm{P}\right)+\mathrm{M} \rightarrow \mathrm{O}\left(2 \mathrm{p}^{43} \mathrm{P}\right)+\mathrm{M}$, has been measured in low pressure DC glow discharges in $\mathrm{O}_{2}$ and in $\mathrm{CO}_{2}$ from the analysis of the temporal decay of fluorescence signals. It has been found to have very similar values in the different mixtures, which reinforces our 
assumption of assuming the same rate coefficient for different collision partners. Moreover, we have assumed the same rate coefficient for reactions Q1 and Q2, due to the similarity of the states involved.

- For the quenching reactions of $\mathrm{O}\left(2 \mathrm{p}^{4}{ }^{1} \mathrm{D}\right)$ and $\mathrm{O}\left(2 \mathrm{p}^{4}{ }^{1} \mathrm{~S}\right)$ (Q3-Q8), the rate coefficients used in the model of Gordiets et al (1995) are taken. The coefficients for collision partners $\mathrm{CO}$ and $\mathrm{CO}_{2}$ are considered as being the same as for $\mathrm{O}_{2}$, according to the same reasoning followed for Q1. When $\mathrm{C}$ is the collision partner, the rate coefficients for $\mathrm{N}$ or $\mathrm{N}_{2}$ quenchers is taken. We should notice that $\mathrm{C}$ has a low molar fraction in the current study, when compared to the other neutrals.

- The quenching rate coefficients of the higher metastable $\mathrm{O}\left(3 \mathrm{~s}^{5} \mathrm{~S}^{0}\right)(\mathrm{Q} 9-\mathrm{Q} 13)$ are taken from Mori et al (1992), where they have been measured for $\mathrm{O}_{2}, \mathrm{CO}, \mathrm{CO}_{2}$ and $\mathrm{N}_{2}$ quenchers from absorption decay curves. For $\mathrm{O}$ and $\mathrm{C}$ quenchers, the coefficients from $\mathrm{O}_{2}$ and $\mathrm{N}_{2}$ are considered, respectively. Furthermore, we assume the rate coefficients for the remaining metastable $\left(3 \mathrm{~s}^{3} \mathrm{~S}^{0}\right)$ to be equal to those for $\mathrm{O}\left(3 \mathrm{~s}^{5} \mathrm{~S}^{0}\right)$, as in Caplinger and Perram (2020).

- Finally, for the quenching of the radiative states (Q14-Q16), the quenching coefficients with $\mathrm{O}_{2}$ are considered, measured for $\mathrm{O}\left(3 \mathrm{p}{ }^{5} \mathrm{P}\right)$ in Dagdigian et al (1988) and for $\mathrm{O}\left(3 \mathrm{p}{ }^{3} \mathrm{P}\right)$ in Niemi et al (2001). As for the other quenching reactions, we assume the same rate coefficient for $\mathrm{O}_{2}$ quencher and for $\mathrm{CO}_{2}, \mathrm{CO}$ and $\mathrm{O}$ quenchers. For $\mathrm{C}$ quencher, the coefficient with $\mathrm{N}_{2}$ quencher measured in Niemi et al (2001) is taken for all radiative states.

- A simple sensitivity analysis study has been performed on the quenching rate coefficients. Simulations have been run while replacing a few quenching coefficients by the corresponding ones from different sets (Dagdigian et al 1988, Pietanza et al 2020, Fiebrandt et al 2020). It has been verified that this leads to no relevant difference in the simulation results in this work, which suggests that an eventual temperature dependence of these coefficients would not affect the conclusions obtained in this work.

\section{Results}

In this section, we apply the model previously described to the different plasma conditions $\mathrm{A}, \mathrm{B}$ and $\mathrm{C}$ explained in section 2.2. Firstly, the initial assumptions of $n_{\mathrm{e}}(r)$ are used. The relationship between $n_{\mathrm{e}}(r)$ and $I_{777}(r)$ is obtained from simulation results and used to correct the input profile of $n_{\mathrm{e}}(r)$ consistently with the measured Gaussian profile of $I_{777}(r)$. This process is explained in detail for the low-confinement discharge of condition A, for which several spatially-resolved discharge parameters are provided from the simulation results. Then, the same procedure is applied to the high-confinement discharge of condition $\mathrm{B}$ and to the wider parameter range of set $\mathrm{C}$. In the case of set $\mathrm{C}$, it is shown that the findings from this study have a direct impact on the flow simulations that provide input $T_{\mathrm{g}}$

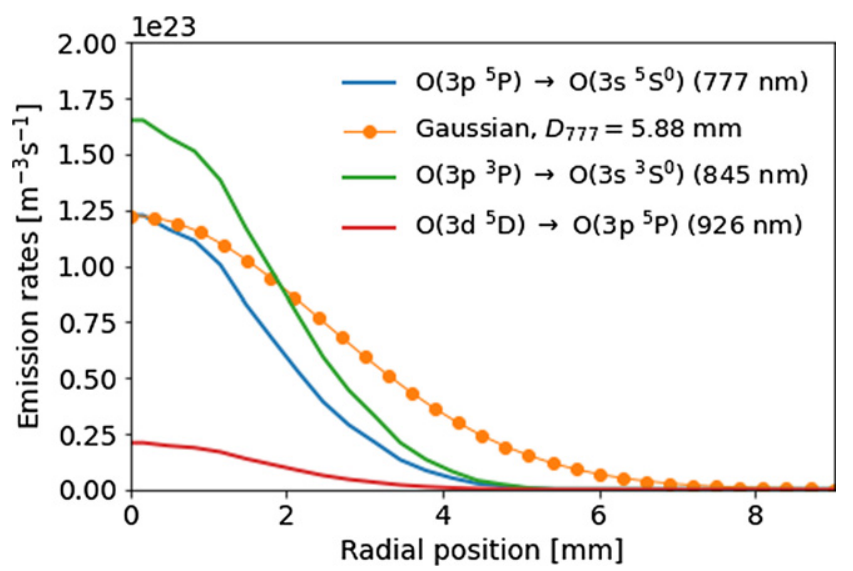

Figure 4. Radial profiles of the simulated emission rates corresponding to the radiative transitions of wavelength 777,845 and $926 \mathrm{~nm}$, for condition A (950 W, 9 slm and $100 \mathrm{mbar}$ ). A Gaussian curve with the measured $\Lambda_{777}$ is also presented.

and composition, and hence the flow model and the procedure to correct spatial profiles of discharge parameters are interdependent. Overall, this study allows both to quantify the impact of parameter changes in the relationships between discharge parameters, and to generalise certain conclusions about the spatial resolution of those parameters and its potential impacts on the study of plasmas for $\mathrm{CO}_{2}$ conversion.

\subsection{Condition A: low-confinement discharge}

4.1.1. Using initial assumptions. We start by using the plasma model to study the low-confinement discharge of condition A, described in section 2.2. The assumption that $n_{\mathrm{e}}(r)$ follows the same Gaussian profile as the measured $I_{777}(r)$ (equation (8)) is taken as a starting point for the model input. Figure 4 depicts the emission rates of the three transitions identified in experiments (R1-R3 in table 3 ), obtained as simulation results, which have the same FWHM as the corresponding emission intensities. A Gaussian profile with the same peak as the simulated $777 \mathrm{~nm}$ emission rate and with the measured FWHM, $\Lambda_{777}=5.88 \mathrm{~mm}$, is added, for comparison with the simulation results.

The results in figure 4 show that the numerically-obtained emission rates have seemingly Gaussian radial profiles, as in the experiments. Moreover, the ratios between the three emission rates agree qualitatively with experimental measurements. However, it is visible that the simulated emission rate of the $777 \mathrm{~nm}$ transition has a narrower radial profile than the measured emission intensity. This result suggests that the initial assumption on input $n_{\mathrm{e}}(r)$ (linearly proportional to $I_{777}(r)$ ) is incorrect. Indeed, the input taken from equation (8) assumes that $n_{\mathrm{e}}$ and $I_{777}$ are linearly proportional. In figure 5 , the dependence of the three considered emission rates on $n_{\mathrm{e}}$ is presented. The simulation results have been fitted in order to derive the proportionality parameter $s$ from equation (5), and we found the best fits to result from polynomial functions of second order. The fits are included in figure 5. According to these fits, the emission rates of the 777, 845 and $926 \mathrm{~nm}$ transitions, 


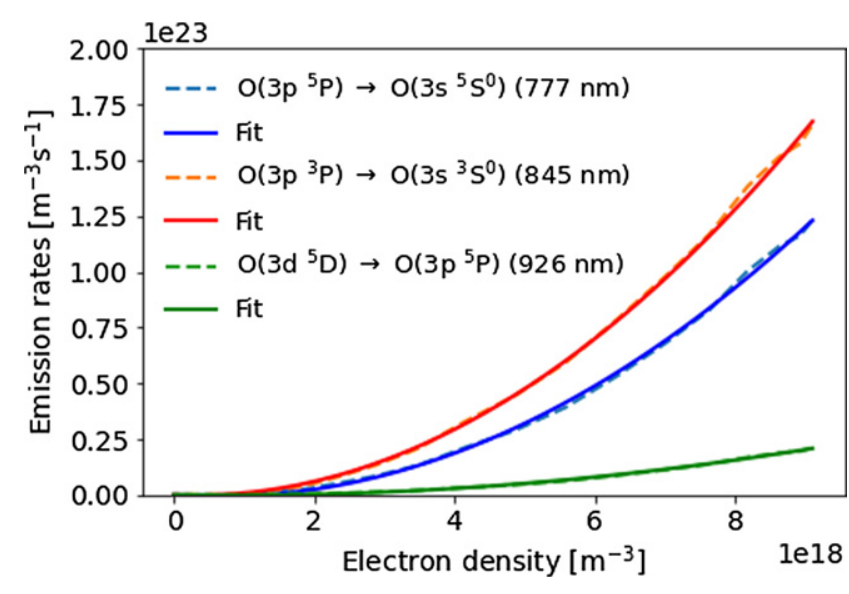

Figure 5. Emission rates corresponding to the radiative transitions of wavelength 777, 845 and $926 \mathrm{~nm}$, as function of $n_{\mathrm{e}}$, for condition A (950 W, $9 \mathrm{slm}$ and $110 \mathrm{mbar})$. Simulation results and polynomial fits.

respectively, $R_{777}, R_{845}$ and $R_{926}$, are given in $\mathrm{m}^{-3} \mathrm{~s}^{-1}$ by:

$$
\begin{aligned}
R_{777}\left(n_{\mathrm{e}}\right)= & -2259\left(\mathrm{~s}^{-1}\right) \times n_{\mathrm{e}}\left(\mathrm{m}^{-3}\right) \\
& +1.731 \times 10^{-15}\left(\mathrm{~m}^{3} \mathrm{~s}^{-1}\right) \times n_{\mathrm{e}}^{2}\left(\mathrm{~m}^{-6}\right) \\
R_{845}\left(n_{\mathrm{e}}\right)= & -1308\left(\mathrm{~s}^{-1}\right) \times n_{\mathrm{e}}\left(\mathrm{m}^{-3}\right) \\
& +2.161 \times 10^{-15}\left(\mathrm{~m}^{3} \mathrm{~s}^{-1}\right) \times n_{\mathrm{e}}^{2}\left(\mathrm{~m}^{-6}\right) \\
R_{926}\left(n_{\mathrm{e}}\right)= & -516.5\left(\mathrm{~s}^{-1}\right) \times n_{\mathrm{e}}\left(\mathrm{m}^{-3}\right) \\
& +3.076 \times 10^{-16}\left(\mathrm{~m}^{3} \mathrm{~s}^{-1}\right) \times n_{\mathrm{e}}^{2}\left(\mathrm{~m}^{-6}\right) .
\end{aligned}
$$

Figure 5 shows that the polynomial functions provide good fits to the simulation results. Furthermore, it is demonstrated that the dependence of the emission rates on $n_{\mathrm{e}}$ is not linear, but rather closer to quadratic, and thus the assumption taken on the profile of $n_{\mathrm{e}}(r)$ should be corrected. This dependence will be explained in the next paragraphs, after correcting the input profile of $n_{\mathrm{e}}(r)$.

4.1.2. Corrections to assumptions. The assumption on $n_{\mathrm{e}}(r)$ that is taken as input in the model can be corrected based on the fits shown previously. Indeed, as we have described $R_{777}\left(n_{\mathrm{e}}\right)$ as a polynomial of second order, $n_{\mathrm{e}}$ can also be described as a polynomial of second order of $R_{777}^{1 / 2}$, with fitting parameters $c_{1}$ and $c_{2}$, of the form:

$$
n_{\mathrm{e}}=c_{1} \times R_{777}^{1 / 2}+c_{2} \times R_{777}
$$

By assuming $R_{777}(r)$ to follow a Gaussian profile of FWHM $\Lambda_{777}$ and characteristic width $\sigma_{777}$ (equation (1)), and then lineintegrating equation (27) considering the measured $n_{\mathrm{e} \Lambda}$, we obtain:

$$
n_{\mathrm{e} \Lambda}=c_{1} \times R_{777}^{1 / 2}(0) \sqrt{4 \pi} \sigma_{777}+c_{2} \times R_{777}(0) \sqrt{2 \pi} \sigma_{777} .
$$

$R_{777}(0)$ can be directly obtained from this equation. Then, equation (27) yields a new profile for $n_{\mathrm{e}}(r)$, that consists of a superposition of two Gaussian curves of different FWHM and that stems directly from the simulation results using the previous assumption for $n_{\mathrm{e}}(r)$. We should notice that $n_{\mathrm{e} 0}$ of the new profile is different than the one firstly assumed, but the measured line-integral $n_{\mathrm{e} \Lambda}$ is conserved. In the case of condition A using the initial assumption for $n_{\mathrm{e}}(r)$, the simulation results yield $c_{1}=3.097 \times 10^{7} \mathrm{~m}^{-3 / 2} \mathrm{~s}^{1 / 2}$ and $c_{2}=-1.861 \times 10^{-5}$ s. Using these values, a new profile of $n_{\mathrm{e}}(r)$ is used as input for the simulations. Then, as the second iteration of simulation results does not yet provide an agreement of the $R_{777}(r)$ profile with experiments within $5 \%$, this procedure is repeated until that condition is satisfied. For the case of condition A, 3 simulations were required to find a satisfying $R_{777}(r)$ profile. The succeeding $n_{\mathrm{e}}(r)$ and $R_{777}(r)$ profiles are presented in figure 6 .

As we can notice from figure 6 , the applied correction procedure results in a broadening of the input $n_{\mathrm{e}}(r)$ and of the output $R_{777}(r)$, until the emission rate approximately matches a Gaussian curve with FWHM of $\Lambda_{777}$, the one measured for emission intensity. This procedure is proven to successfully reproduce the measured radial profile of $R_{777}$. As such, this model can radially resolve discharge parameters. In fact, the $n_{\mathrm{e}}(r)$ profile that matches the measured output can be approximately described as a Gaussian profile with FWHM = 9.93 $\mathrm{mm}$, i.e. with an FWHM value 1.69 times higher than the one of $R_{777}$. Consequently, as $n_{\mathrm{e} \Lambda}$ is conserved in equation (6), the peak $n_{\mathrm{e} 0}$ is lower than initially assumed, by approximately the same factor of 1.69. Following equation (2), the proportionality parameter $s$ is calculated as $1.69^{2} \simeq 2.85$, close to the stated upper limit of 2 . The fact that $n_{\mathrm{e}}(r)$ follows a seemingly Gaussian profile agrees with previous studies of contracted discharges at intermediate to high pressures in Petrov and Ferreira (1999), Martinez et al (2004), Kabouzi et al (2007), Dyatko et al (2008), Golubovskii et al (2011), Shneider et al (2014), Golubovskii et al (2017), Ridenti et al (2018), Zhong et al (2019). Furthermore, we find $n_{\mathrm{e}}$ to have a broader radial profile than the emission intensity, which consists in the phenomenon of optical contraction reported in Golubovskii et al $(2011,2020)$. It is also visible in figure 6 that, besides the Gaussian fit, $n_{\mathrm{e}}(r)$ can also be fitted by a Bessel function or by a paraboloidal function (not shown), in agreement with the radially-resolved $n_{\mathrm{e}}$ Thomson scattering measurements in the argon MW discharge in Carbone et al (2012). However, unlike the Gaussian fit, these functions become negative well before reaching the walls, which is not compatible with the presence of diffusive transport. As such, we deem it more fit to describe the radial profiles of $n_{\mathrm{e}}$ as Gaussian functions.

4.1.3. Kinetics of $O$ emission intensity. To understand the proportionality parameter $s$ around 2.85, the main reaction rates of production and destruction of the upper state of the 777 $\mathrm{nm}$ transition, $\mathrm{O}\left(3 \mathrm{p}{ }^{5} \mathrm{P}\right)$, are presented in figure 7 , from the simulation results of iteration 3 .

Figure 7 shows that the main production reactions of $\mathrm{O}\left(3 \mathrm{p}^{5} \mathrm{P}\right)$ are electron-impact reactions, firstly through electron impact with the ground-state of atomic oxygen, and then through collisions with the higher metastables $\mathrm{O}\left(3 \mathrm{~s}{ }^{5} \mathrm{~S}^{0}\right)$ and $\mathrm{O}\left(3 \mathrm{~s}^{3} \mathrm{~S}^{0}\right)$. This result highlights the relevance of stepwise excitation put forward in Caplinger and Perram (2020) and shown for $\mathrm{O}\left(3 \mathrm{p}^{3} \mathrm{P}\right)$ in Fiebrandt et al (2020). Quenching from upper 

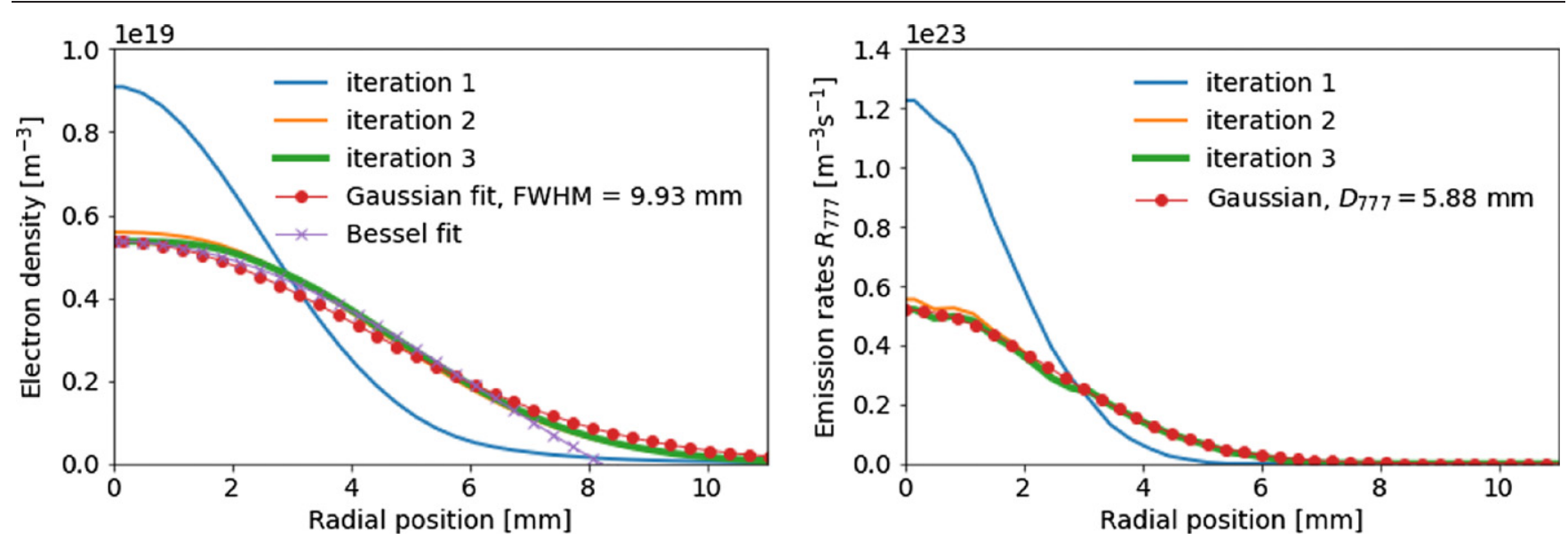

Figure 6. Radial profiles of $n_{\mathrm{e}}$ (on the left) and $R_{777}$ (on the right) for successive iterations, for condition $\mathrm{A}$ ( $950 \mathrm{~W}, 9 \mathrm{slm}$ and $110 \mathrm{mbar}$ ). Each iteration corresponds to a different input $n_{\mathrm{e}}(r)$. A Gaussian function and a Bessel function have been fitted to the last profile of $n_{\mathrm{e}}(r)$. A Gaussian curve with experimentally-obtained $\Lambda_{777}$ is also compared to the last result of $R_{777}(r)$.

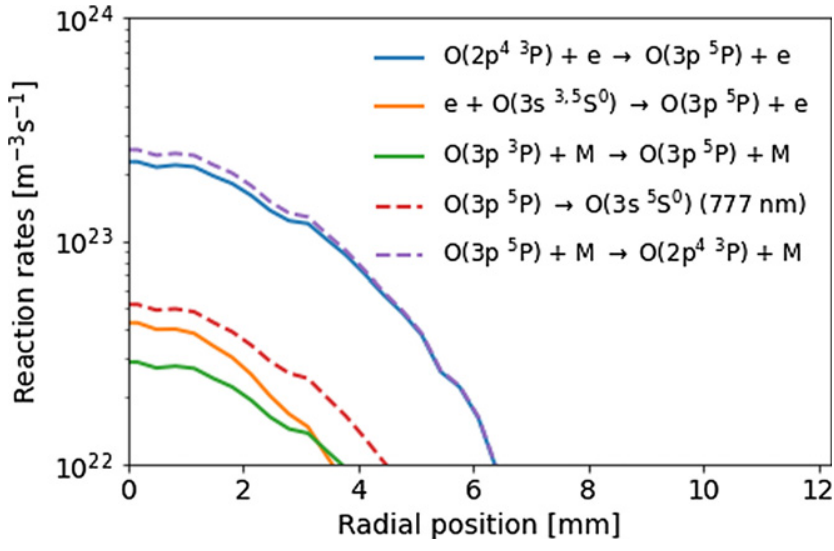

Figure 7. Radial profiles of the main rates of source and loss of the upper radiative state of the $777 \mathrm{~nm}$ transition, $\mathrm{O}\left(3 \mathrm{p}{ }^{5} \mathrm{P}\right)$, for condition A ( $950 \mathrm{~W}, 9$ slm and 110 mbar). Production rates in solid lines and loss rates in dashed lines. Simulation results from iteration 3 .

states (reaction Q1 in table 4) and emission from upper states (R3, not shown in figure 7) also populate $\mathrm{O}\left(3 \mathrm{p}{ }^{5} \mathrm{P}\right)$, although this last reaction contributes to less than $5 \%$ of production. Therefore, cascade emission populating the upper states also influences the production of $\mathrm{O}\left(3 \mathrm{p}{ }^{5} \mathrm{P}\right)$, as highlighted in both Fiebrandt et al (2020) and Caplinger and Perram (2020). Other production mechanisms of $\mathrm{O}\left(3 \mathrm{p}^{5} \mathrm{P}\right)$ that have been considered in the simulations, such as dissociative excitation and other electron-impact reactions, appear to play a negligible role. As far as losses are concerned, the main mechanisms are quenching and radiative emission. Radiation self-absorption has a role in these simulations, confirming the importance attributed to it in Fiebrandt et al (2020). Indeed, the emission coefficient $\tau_{\text {rad }}^{-1}$ of the $777 \mathrm{~nm}$ transition in condition $\mathrm{A}$ is a function of $r . \tau_{\text {rad }}^{-1}$ is $3.38 \times 10^{7} \mathrm{~s}^{-1}$ at $r=0$ and increases to its natural value of $3.69 \times 10^{7} \mathrm{~s}^{-1}$ for $r>5 \mathrm{~mm}$. Other loss processes of $\mathrm{O}\left(3 \mathrm{p}^{5} \mathrm{P}\right)$ included in the model, through transport (convective and diffusive) and electron impact, are shown to have a lesser role for $\mathrm{O}\left(3 \mathrm{p}^{5} \mathrm{P}\right)$ balance. Overall, we can say that hypothesis 1 in section 2.1 on the $777 \mathrm{~nm}$ radiation balance is approximately verified, as it correctly estimates the importance of direct electron-impact excitation, quenching and emission from $\mathrm{O}\left(3 \mathrm{p}{ }^{5} \mathrm{P}\right)$, but neglects the secondary role of stepwise excitation and of the states above $\mathrm{O}\left(3 \mathrm{p}^{5} \mathrm{P}\right)$.

Hypothesis 2 is also approximately verified, although $\tau_{\text {rad }}^{-1}$ is not radially homogeneous. Indeed, quenching is the main loss process $\left(n_{\mathrm{g}} k_{\mathrm{Q}}>\tau_{\text {rad }}^{-1}\right)$ and $k_{\mathrm{Q}}$ is approximately radially homogeneous. Thus, if we consider an effective electron-impact excitation coefficient $k_{\text {eff }}\left(T_{\mathrm{e}}\right)$ including stepwise excitation, we can write:

$$
I_{777} \propto \tau_{\text {rad }}^{-1} n_{\mathrm{e}} x_{\mathrm{O}} k_{\mathrm{eff}}\left(T_{\mathrm{e}}\right)
$$

In the plasma, as we approach the centre, $n_{\mathrm{e}}$ increases and $\tau_{\text {rad }}^{-1}$ slightly decreases. Then, in figure 8 , we evaluate the radial dependence of $T_{\mathrm{e}}$ (2/3 of the electron mean energy), of $E / n_{\mathrm{g}}$ and of the electric field magnitude $E$, as well as the dependence of the electron-impact excitation coefficient on $n_{\mathrm{e}}$. The impact of stepwise excitation is highlighted by depicting both the ground-state electron-impact excitation coefficient to $\mathrm{O}\left(3 \mathrm{p}{ }^{5} \mathrm{P}\right), k_{\mathrm{exc}}$, and the effective coefficient including also excitation from the higher metastables, $k_{\text {eff }}$.

The simulation results in figure 8 indicate that $T_{\mathrm{e}}$ and $E / n_{\mathrm{g}}$ in the $\mathrm{CO}_{2} \mathrm{MW}$ discharge have concave shapes as in the studies of contracted discharges in Kabouzi et al (2007), Gregório et al (2010), Golubovskii et al (2011), Gregório et al (2012), Golubovskii et al (2017), Ridenti et al (2018), Zhong et al (2019), Golubovskii et al (2020), due to the self-reinforcing cycle between heating and electron-driven collisionality. However, here the electric field magnitude presents a rather flat profile in the whole plasma region up to $r=10 \mathrm{~mm}$. We should notice that for higher radial positions, where $n_{\mathrm{e}}(r) \leqslant 7 \times 10^{16} \mathrm{~m}^{-3}$ and no electron-impact ionisation takes place, the simulation result of $E / n_{\mathrm{g}}$ is assumed to have a minimum value of $1 \mathrm{Td}$, which also affects the calculation of $T_{\mathrm{e}}$ and $E$. Conversely, in the 3D EM simulation results in Groen et al (2019), addressing a low-confinement discharge in the same MW reactor and assuming equation (8) as input profile, $E / n_{\mathrm{g}}$ and $E$ have rather flat profiles only in the first $4 \mathrm{~mm}$ near the axis, and then a 

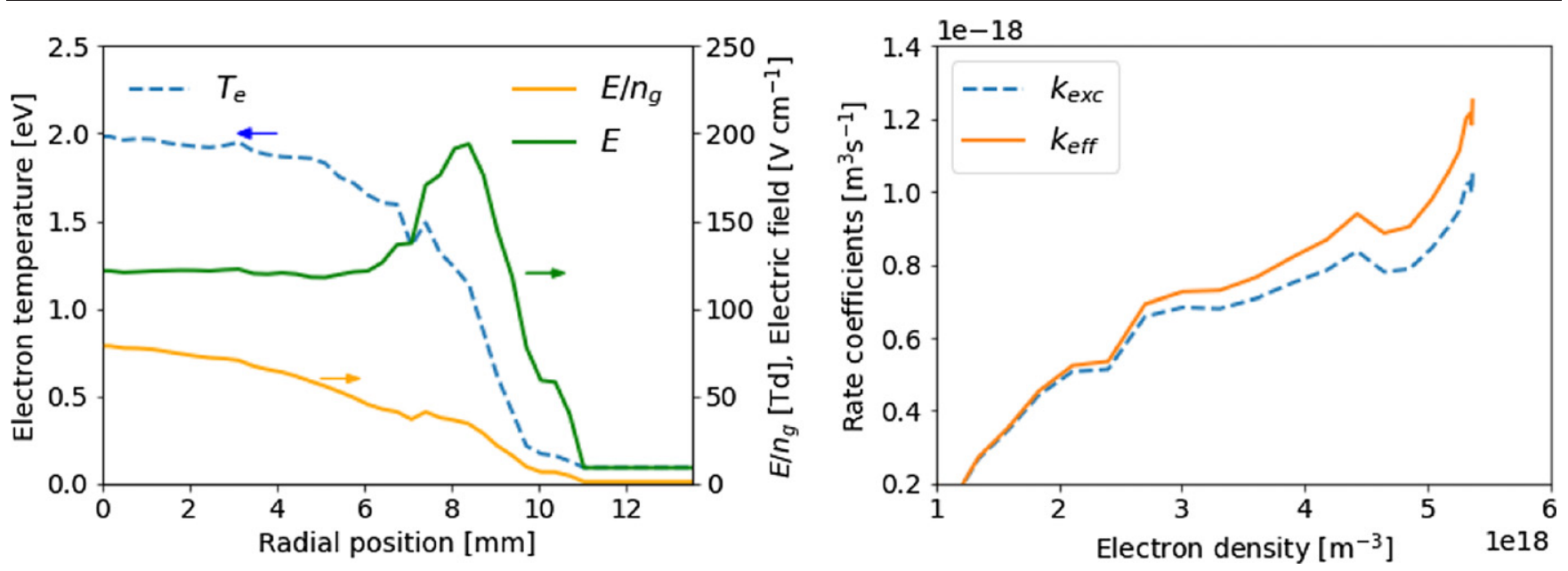

Figure 8. On the left, radial profiles of $T_{\mathrm{e}}, E / n_{\mathrm{g}}$ and $E$. On the right, electron-impact excitation coefficients of $\mathrm{O}\left(3 \mathrm{p}{ }^{5} \mathrm{P}\right)$, as function of $n_{\mathrm{e}}$. $k_{\mathrm{exc}}$ is the coefficient corresponding to excitation from ground-state only. $k_{\mathrm{eff}}$ is an effective coefficient, corresponding to excitation from ground-state $\mathrm{O}\left(2 \mathrm{p}^{4}{ }^{3} \mathrm{P}\right), \mathrm{O}\left(3 \mathrm{~s}{ }^{5} \mathrm{~S}^{0}\right)$ and $\mathrm{O}\left(3 \mathrm{~s}^{3} \mathrm{~S}^{0}\right)$. Simulation results from iteration 3 .

convex shape radially outwards. Moreover, the values of $E / n_{\mathrm{g}}$ and $E$ reported in that work are close to twice as much as those shown here. These discrepancies and the importance of the current study for the results in Groen et al (2019) are discussed in section 4.4.

Figure 8 also shows that, as $E / n_{\mathrm{g}}$ increases towards the axis where $n_{\mathrm{e}}$ is also higher, the electron-impact excitation coefficients increase almost linearly with $n_{\mathrm{e}}$. The increase of $k_{\mathrm{exc}}$ and $k_{\text {eff }}$ is expected in the context of contracted discharges where gas heating and electron-driven collisionality reinforce each other and where the electron-impact ionisation coefficient also needs to increase to maintain a higher $n_{\mathrm{e}}$ (Viegas et al 2020). This relationship means that the main production channels of $\mathrm{O}\left(3 \mathrm{p}{ }^{5} \mathrm{P}\right)$ cannot be considered radially homogeneous, which invalidates hypothesis 3 in section 2.1. It also shows that the concave shape of both $E / n_{\mathrm{g}}$ and $n_{\mathrm{e}}$ is the main reason for the optical contraction phenomenon observed in this work, which is in agreement with the analysis reported in Golubovskii et al (2020) concerning helium glow discharges.

The last element to analyse in equation (29) is the molar fraction of atomic oxygen $x_{\mathrm{O}}$, that has been measured and shown to have a concave profile in figure 2 . If we consider a plasma region of $5.1 \mathrm{~mm}$ radius where $n_{\mathrm{e}}$ is halved (figure 6), $x_{\mathrm{O}}$ drops radially in the same region also by approximately a factor 2 (figure 2). Indeed, $n_{\mathrm{e}}$ and $x_{\mathrm{O}}$ have rather similar radial profiles. As assumed in hypothesis $4, x_{\mathrm{O}}$ is defined by thermal chemistry and charged particle kinetics, in addition to a contribution of transport which remains largely unknown in this reactor. Hence, the large radial variations of $T_{\mathrm{g}}$ justify the approximately linear correlation between $n_{\mathrm{e}}$ and $x_{\mathrm{O}}$. This assessment allows to explain, from equation (29), why the proportionality parameter $s$ between $I_{777}$ and $n_{\mathrm{e}}$ is found to be close to 2.85 . As the radial position approaches the centre and $n_{\mathrm{e}}$ increases, although $\tau_{\text {rad }}^{-1}$ slightly decreases, $x_{\mathrm{O}}$ and $k_{\text {eff }}$ increase proportionally to $n_{\mathrm{e}}$, justifying a proportionality parameter between 2 and 3 . We should notice that this conclusion holds despite the experimental uncertainty in input parameters, as the proportionality between emission intensity and $n_{\mathrm{e}}$

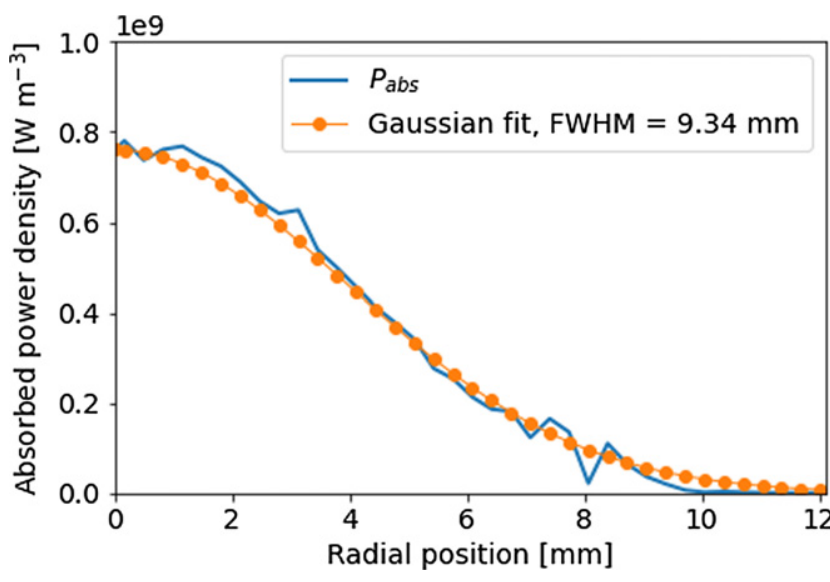

Figure 9. Radial profile of absorbed power density for condition A ( $950 \mathrm{~W}, 9 \mathrm{slm}$ and $110 \mathrm{mbar}$ ) and Gaussian fit. Simulation results from iteration 3 .

is mostly dependent on the shape of radial profiles, which is independent of the absolute value of $n_{\mathrm{e} \Lambda}$ and of the relatively low experimental uncertainties in $T_{\mathrm{g}}$ and composition.

4.1.4. Power density profile. Another very important parameter for MW discharge characterisation that is affected by the optical contraction, due to its proximity to $n_{\mathrm{e}}$, is the absorbed power density, $P_{\text {abs }}$. In figure 9 we assess the radial profile of $P_{\text {abs }}(r)$, calculated according to equation (18).

Figure 9 shows that $P_{\mathrm{abs}}(r)$ can also be approximated as a Gaussian curve for condition A, with an FWHM $\Lambda_{P_{\mathrm{abs}}}=9.34 \mathrm{~mm}$, very close to the one of $n_{\mathrm{e}}$. Indeed, we find an FWHM 1.59 times higher than $\Lambda_{777}$ and thus a proportionality $I_{777} \propto P_{\mathrm{abs}}^{s_{\mathrm{p}}}, s_{\mathrm{p}} \simeq 2.5$. Following equation (18), and considering that the electron transport terms have a weight of only $2 \%$ on the calculation of $P_{\mathrm{abs}}$, we can write $P_{\mathrm{abs}}(r) \simeq\left(\frac{P_{\mathrm{el}}}{n_{\mathrm{g}}}(r)+\frac{P_{\text {inel }}}{n_{\mathrm{g}}}(r)+\frac{P_{\text {growth }}}{n_{\mathrm{g}}}(r)\right) n_{\mathrm{g}}(r) n_{\mathrm{e}}(r)$. We should notice that the collisional electron losses increase with $E / n_{\mathrm{g}}$ and thus also with $n_{\mathrm{e}}$, but $n_{\mathrm{g}}$ is inversely correlated with $n_{\mathrm{e}}$. 


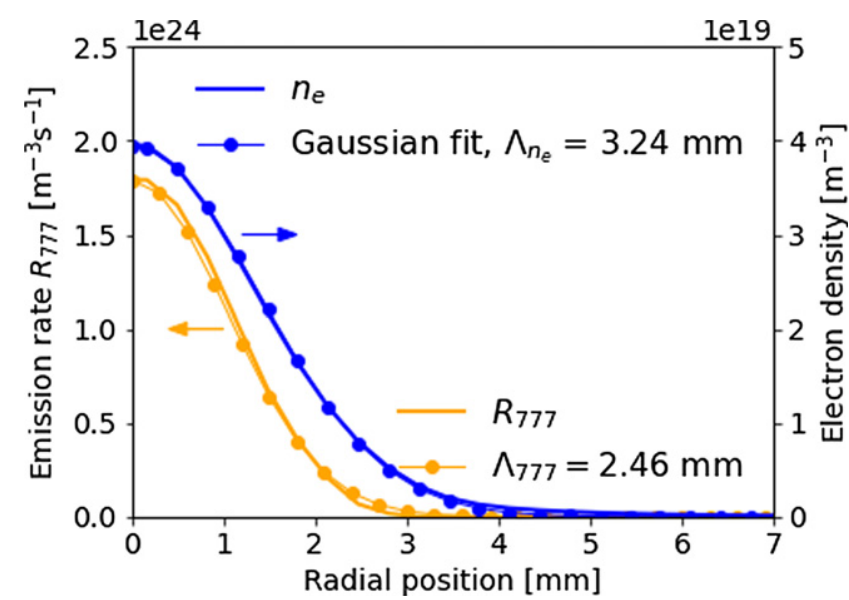

Figure 10. Radial profiles of the $777 \mathrm{~nm}$ transition emission rate (left-side axis) and the corrected electron density (right-side axis) for condition B ( $860 \mathrm{~W}, 12 \mathrm{slm}$ and $150 \mathrm{mbar})$. The simulation results after correction procedure and the Gaussian curves with measured FWHM $\Lambda_{777}$ and fitted FWHM $\Lambda_{n_{\mathrm{e}}}$ are presented.

These relationships justify a similar but lower proportionality parameter between $I_{777}$ and $P_{\mathrm{abs}}$ than between $I_{777}$ and $n_{\mathrm{e}}$ for condition A. The results in this section show that it is relevant to correct the assumptions on the proportionality between $I_{777}$, $n_{\mathrm{e}}$ and $P_{\mathrm{abs}}$. Moreover, it is demonstrated that we can successfully resolve discharge parameters from $I_{777}$ and the proposed procedure, for the case of a low-confinement discharge.

\subsection{Condition B: high-confinement discharge}

The confinement degree of the $\mathrm{CO}_{2} \mathrm{MW}$ discharge depends strongly on input power and pressure (Wolf et al 2020b). In this section, we evaluate whether the conclusions taken from the study of condition A can be extended to conditions of highconfinement discharges. Starting from the input profile of $n_{\mathrm{e}}(r)$ described in section 2.2, the same correction procedure used for condition A is applied to condition B. After one correction, the $R_{777}(r)$ and $n_{\mathrm{e}}(r)$ profiles represented in figure 10 are obtained. Two Gaussian curves are added to the figure: one with the simulated peak of $R_{777}$ and the measured FWHM $\Lambda_{777}$, and one as a fit to the $n_{\mathrm{e}}(r)$ profile.

Similarly to condition A, figure 10 shows that an approximately Gaussian $R_{777}$ radial profile is obtained with the experimental $\Lambda_{777}$ as FWHM, in the high-confinement condition B. Indeed, the correction procedure we developed is successful in reproducing the experimental profiles for different conditions of power, pressure and confinement degree. The radial profile of $n_{\mathrm{e}}$ is obtained as a Gaussian curve of FWHM $\Lambda_{n_{\mathrm{e}}}>\Lambda_{777}$, which confirms the phenomenon of optical contraction. The ratio $\Lambda_{n_{\mathrm{e}}} / \Lambda_{777}$ for condition B is 1.32 and the proportionality parameter $s$ between $n_{\mathrm{e}}$ and $I_{777}\left(I_{777} \propto n_{\mathrm{e}}^{s}\right)$ is 1.74 , lower than the corresponding values for condition $\mathrm{A}$ (1.69 and 2.85, respectively). The relationship between $n_{\mathrm{e}}$ and $I_{777}$ is also described by equation (29) in condition B, as the main reaction mechanisms for the balance of $\mathrm{O}\left(3 p{ }^{5} \mathrm{P}\right)$ are the same as for condition A. However, we should notice that stepwise excitation to $\mathrm{O}\left(3 \mathrm{p}{ }^{5} \mathrm{P}\right)$ has a more important role in

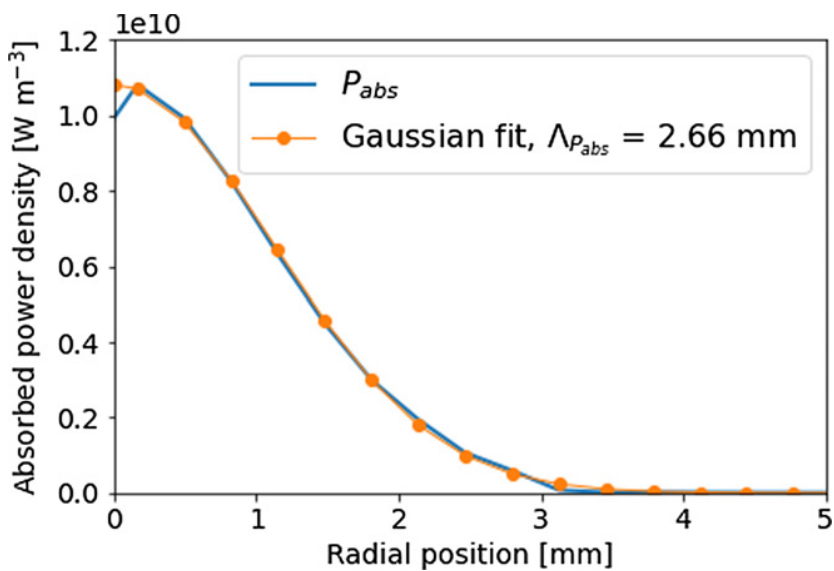

Figure 11. Radial profile of simulated absorbed power density for condition B (860 W, 12 slm and 150 mbar) and Gaussian fit.

high-confinement conditions. Following equation (29), the difference in $s$ between conditions A and B is justified by the different behaviour of $\tau_{\text {rad }}^{-1}$ and of $x_{\mathrm{O}}$ between these discharges. In fact, the electron-impact excitation coefficient to $\mathrm{O}\left(3 \mathrm{p}{ }^{5} \mathrm{P}\right)$ is approximately linearly proportional to $n_{\mathrm{e}}$ in both conditions $\mathrm{A}$ and B. $T_{\mathrm{e}}$ and $E / n_{\mathrm{g}}$ are concave and $E$ is approximately flat within the plasma in both contracted modes. In the case of condition $\mathrm{B}, T_{\mathrm{e}}$ and $E / n_{\mathrm{g}}$ have maxima of $2.4 \mathrm{eV}$ and 110 $\mathrm{Td}$, and $E \simeq 140 \mathrm{~V} \mathrm{~cm}^{-1}$ for $r<3 \mathrm{~mm}$, higher values than for condition A. Contrarily to the low-confinement discharge, these values should not be compared to those found for a high-confinement discharge in Groen et al (2019) since significantly higher pressure and lower temperature are considered in that work. The lower proportionality parameter $s$ in the highconfinement condition is justified mostly by the more homogeneous $x_{\mathrm{O}}$ profile revealed in the measurements presented in figure 3. $x_{\mathrm{O}}$ is close to 0.4 for $r<3 \mathrm{~mm}$ and decreases to 0.2 at $r=5 \mathrm{~mm}$, while $n_{\mathrm{e}}$ decreases by two orders of magnitude along the same distance. Moreover, the emission coefficient $\tau_{\text {rad }}^{-1}$ evolves inversely to $n_{\mathrm{e}}$, as it falls linearly from its original value of $3.69 \times 10^{7} \mathrm{~s}^{-1}$ for $r>4 \mathrm{~mm}$ to $2 \times 10^{7} \mathrm{~s}^{-1}$ at $r=0$, due to the higher self-absorption in high-confinement conditions. Finally, the simulated power density is also fitted to a Gaussian curve, as shown in figure 11.

Figure 11 shows that the Gaussian curve describing $P_{\mathrm{abs}}(r)$ for condition $\mathrm{B}$ has $\Lambda_{P_{\mathrm{abs}}}=2.66 \mathrm{~mm}$, very close to $\Lambda_{777}$. Indeed, we find $\Lambda_{P_{\mathrm{abs}}} / \Lambda_{777}=1.08$ and thus $s_{\mathrm{p}}=1.16$, very close to the initial assumption of $s_{\mathrm{p}}=1$ (hypothesis 6 in section 2.1). Furthermore, figure 9 shows a dip in the simulated $P_{\mathrm{abs}}(r)$ in the axis of the discharge. As noted in the study of condition $\mathrm{A}$, following the electron energy equation (equation (18)), collisional electron losses increase with $E / n_{\mathrm{g}}$ and thus also with $n_{\mathrm{e}}$, but $n_{\mathrm{g}}$ is inversely correlated with $n_{\mathrm{e}}$. The sharp gradients of $T_{\mathrm{g}}$ and $n_{\mathrm{g}}$ in high-confinement conditions help explain both the dip in $P_{\text {abs }}$ and $\Lambda_{P_{\text {abs }}} \ll \Lambda_{n_{\mathrm{e}}}$.

The conclusions from the study of condition A on the most relevant factors to take into account to relate $I_{777}$ and $n_{\mathrm{e}}$ are kept when changing power, pressure and confinement degree in condition B. However, it is shown here that the proportionality between $I_{777}, n_{\mathrm{e}}$ and $P_{\mathrm{abs}}$ is not the same in every plasma, 
as $n_{\mathrm{e}}, T_{\mathrm{g}}$ and $x_{\mathrm{O}}$ have significantly different profiles according to the confinement degree. Hence, different plasma conditions require dedicated analysis in order to spatially resolve discharge parameters.

\subsection{Set of conditions C: interdependence with flow modelling}

In the set of conditions $\mathrm{C}$, described in section 2.2, the input of $T_{\mathrm{g}}$ and composition used to resolve discharge parameters is obtained from flow simulations of the model described in Wolf et al (2020a). The observed confinement degree in these conditions generally stands between the one of the low-confinement discharge of condition A and that of the high-confinement discharge of condition $\mathrm{B}$. The values and extension of $T_{\mathrm{g}}$ and dissociation degree are also in between those of the two previously studied conditions. As such, by applying the same correction procedure as in the previous sections, we expect to obtain, for the set of conditions $\mathrm{C}$, spatial profiles and proportionality parameters $s$ and $s_{\mathrm{p}}$ in between those found for conditions A and B. Indeed, the Gaussian radial profiles of $R_{777}$ with experimentally-measured $\Lambda_{777}$ have been obtained for this set of conditions with 3 or 4 iterations of the assumed $n_{\mathrm{e}}(r)$ profiles. As a result, corrected profiles of $n_{\mathrm{e}}(r)$ and $P_{\mathrm{abs}}(r)$ have been retrieved. While $n_{\mathrm{e}}(r)$ is well described by a single Gaussian curve, $P_{\text {abs }}(r)$ for the set of conditions $C$ slightly deviates from this shape, due to the dip at the axis that has been discussed for condition $\mathrm{B}$, and whose depth increases with pressure. Still, the profiles $P_{\text {abs }}(r)$ have been fitted to Gaussian functions with FWHM $\Lambda_{P_{\mathrm{abs}}}$. In figure $12, \Lambda_{777}, \Lambda_{n_{\mathrm{e}}}$ and $\Lambda_{P_{\mathrm{abs}}}$ are compared as function of pressure, and the ratios between these quantities are represented for the set of conditions $\mathrm{C}$.

Figure 12 shows that $\Lambda_{n_{\mathrm{e}}}$ and $\Lambda_{P_{\mathrm{abs}}}$ are, as $\Lambda_{777}$, approximately independent of pressure for pressures above $150 \mathrm{mbar}$, confirming the conclusion from Wolf et al (2019) that $\Lambda_{n_{\mathrm{e}}}$ is independent of pressure in H-mode conditions. Both $\Lambda_{n_{\mathrm{e}}}$ and $\Lambda_{P_{\text {abs }}}$ are higher than $\Lambda_{777}$, as the discharge is in every case broader than initially assumed. For the set of conditions $\mathrm{C}$, $\Lambda_{n_{\mathrm{e}}} / \Lambda_{777}$ varies between 1.45 and 1.73 and $\Lambda_{P_{\text {abs }}} / \Lambda_{777}$ stands between 1.42 and 1.58 . We should notice that the correction procedure leads to a decrease of $n_{\mathrm{e} 0}$ relative to the initial assumption in the same proportion as the increase in $\Lambda_{n_{\mathrm{e}}}$. $P_{\text {abs0 }}$ also decreases with the correction procedure, but in a different proportion with respect to $\Lambda_{P_{\mathrm{abs}}}$, since it is a simulation result and no line-integrated conservation is imposed as in the case of $n_{\mathrm{e}}$. In fact, $P_{\mathrm{abs} 0}$ decreases by a factor between 1.6 (for 296 mbar) and 4.7 (for $108 \mathrm{mbar}$ ). The values of the ratios $\Lambda_{n_{\mathrm{e}}} / \Lambda_{777}$ and $\Lambda_{P_{\mathrm{abs}}} / \Lambda_{777}$ are very close to those found in the case of the low-confinement discharge in condition $\mathrm{A}$ as, following equation (29), $x_{\mathrm{O}}$ and $k_{\text {eff }}$ are approximately linearly proportional to $n_{\mathrm{e}}$, and $\tau_{\text {rad }}^{-1}$ is convex in the plasma region. We should notice that the $x_{\mathrm{O}}(r)$ profile is a direct input from 2D flow simulations in the set of conditions C. In turn, the flow model takes the $n_{\mathrm{e}}(r)$ and $P_{\text {abs }}(r)$ profiles as input. This has been done firstly using the assumptions from equations (8) and (9), i.e.i.e. $\Lambda_{P_{\mathrm{abs}}}=\Lambda_{n_{\mathrm{e}}}=\Lambda_{777}$. In this work, the $n_{\mathrm{e}}(r)$ and $P_{\text {abs }}(r)$ profiles are corrected for the 7 cases of the set of conditions $\mathrm{C}$, using the ratios found here. Indeed, these profiles are taken with approximately 1.6 times higher radial and axial
FWHM, and used as input in the model described in Wolf $e t$ al (2020a). As $n_{\mathrm{e}}$ is conserved line-integrated and $P_{\mathrm{abs}}$ is conserved volume integrated, their peaks are, respectively, around 1.6 and 4 times lower. Different simulation results are obtained with these inputs, with respect to the initial ones using the assumptions from equation (8) and (9). To show the resulting difference, the initial and corrected radial profiles of $T_{\mathrm{g}}$ and $x_{\mathrm{O}}$ at the axial position of highest $T_{\mathrm{g}}$ are represented in figure 13 for 121 mbar and 296 mbar. Besides the importance of $x_{\mathrm{O}}$ and $T_{\mathrm{g}}$ for the study of spatial profiles of discharge parameters, we have seen in figures 2 and 3 that $T_{\mathrm{g}}$ and $x_{\mathrm{O}}$ are correlated with the production of $\mathrm{CO}$, the dissociation product that these reactors are aimed at producing.

In the flow model in Wolf et al (2020a), the peak gas temperature is set to the one measured in Doppler broadening measurements (Wolf et al 2019). As such, the broader profile of $P_{\text {abs }}$ leads to the same peak temperature but a slightly broader high-temperature profile, as shown in figure 13. Although apparently small, this broadening increases thermally-driven dissociation in the plasma and hence produces higher and more extended profiles of $\mathrm{O}$ and $\mathrm{CO}$ molar fractions, as shown on the right side of the same figure. This effect is reinforced by the broadening of the $n_{\mathrm{e}}(r)$ profile driving electron-impact dissociation reactions.

The changes in temperature and composition have an influence on the study of spatial profiles undergone in the current work, and the two models (flow and plasma) are therefore interdependent. The plasma simulations are run for the corrected cases of the set of conditions $C$ to verify the consistency of our results. For $p<150 \mathrm{mbar}$, the dependence between $I_{777}$, $n_{\mathrm{e}}$ and $P_{\mathrm{abs}}$ is very similar with or without this correction, as the corrected $x_{\mathrm{O}}$ has higher values but a similar radial profile as in the initial assumption. However, for highly-contracted conditions with $p>150$ mbar, the correction leads to a more flat profile of $x_{\mathrm{O}}(r)$, similar to the one presented in figure 3 , and therefore to a dependence between the studied parameters that is closer to that of condition B. As such, in those cases the interdependence between the inputs of the two models is not fully consistent. We can only conclude that the values of the ratios $\Lambda_{n_{\mathrm{e}}} / \Lambda_{777}$ and $\Lambda_{P_{\mathrm{abs}}} / \Lambda_{777}$ stand between those of condition $\mathrm{B}$, respectively 1.32 and 1.08 , and those of figure 12 , both close to 1.6. Although allowing for the study of a wider parameter range, the interdependence between the two models presents some limitations with respect to using the plasma model with experimentally measured input. Those limitations can be overcome through the development of a self-consistent model simulating flow, temperature, composition and plasma parameters.

Gathering the results of conditions $\mathrm{A}, \mathrm{B}$ and $\mathrm{C}$, we can say that the proportionality between $n_{\mathrm{e}}, P_{\mathrm{abs}}$ and $I_{777}$ can be described as $I_{777} \propto n_{\mathrm{e}}^{s}, 1.82 \leqslant s \leqslant 2.94$ and $I_{777} \propto$ $P_{\mathrm{abs}}^{s_{\mathrm{p}}}, 1.22 \leqslant s_{\mathrm{p}} \leqslant 2.50$. The disparity between these proportionality parameters shows that the spatial resolution of discharge parameters from emission intensity is rather complex and dependent on particular conditions. As such, spatiallyresolved measurements and simulations are two essential and complementary tools to accurately describe the spatial profiles of discharge parameters in contracted plasmas. Moreover, we 

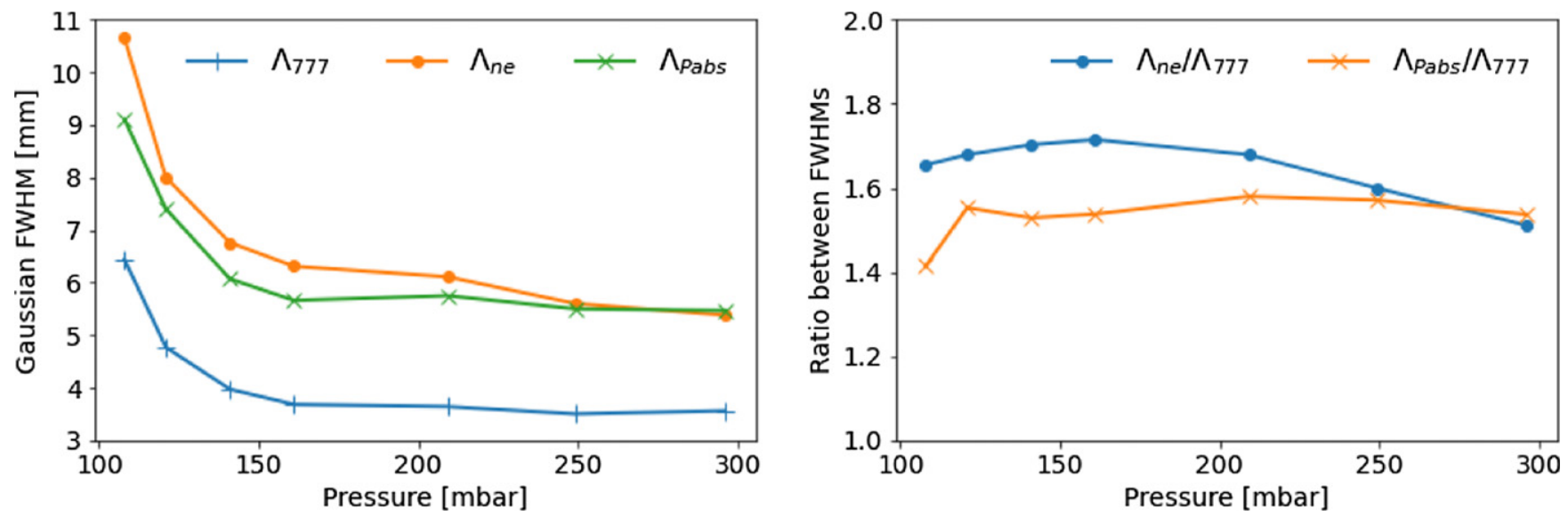

Figure 12. On the left, different diameters as function of pressure, after applying the correction procedure. On the right, the ratios between $\Lambda_{n_{\mathrm{e}}}$ and $\Lambda_{777}$ and between $\Lambda_{P_{\mathrm{abs}}}$ and $\Lambda_{777}$, as function of pressure. Results obtained for the set of conditions C.
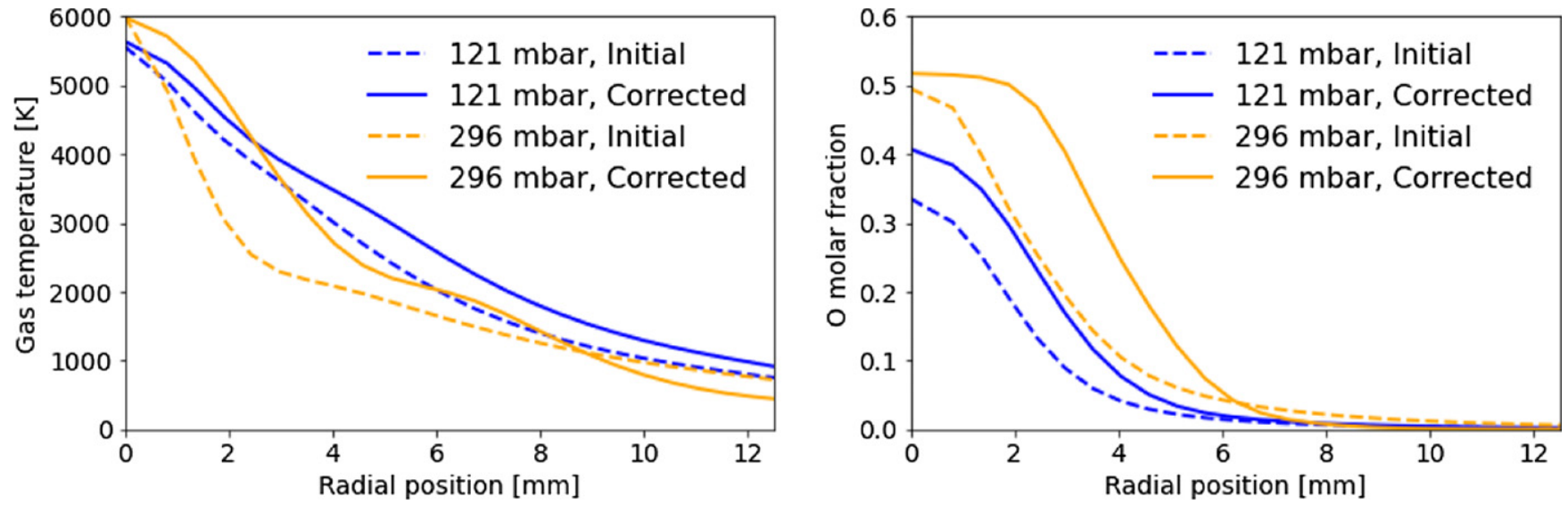

Figure 13. Radial profiles of flow model results, using the initial assumptions on $n_{\mathrm{e}}(r)$ and $P_{\text {abs }}(r)\left(\Lambda_{P_{\mathrm{abs}}}=\Lambda_{n_{\mathrm{e}}}=\Lambda_{777}\right)$, and the corrected $n_{\mathrm{e}}(r)$ and $P_{\text {abs }}(r)$ profiles with $\Lambda_{P_{\text {abs }}} \sim \Lambda_{n_{\mathrm{e}}} \sim 1.6 \times \Lambda_{777}$. Gas temperature is represented on the left and $\mathrm{O}$ molar fraction on the right.

should notice that, despite the complexity, $\Lambda_{n_{\mathrm{e}}}$ and $\Lambda_{P_{\text {abs }}}$ are around 1.6 times higher than $\Lambda_{777}$ for $p<150$ mbar and the values of the proportionality parameters $s$ and $s_{\mathrm{p}}$ are close to 2.5. Hence, these values can be assumed for $\mathrm{MW} \mathrm{CO}_{2}$ contracted plasmas that are not fully contracted, i.e. whose contraction regime is not $\mathrm{H}$-mode according to the characterisation in Wolf et al (2020b). Based on this modelling study, our evaluation of the hypotheses listed in section 2.1 is summarised in table 5 .

\subsection{Discussion on implications of the correction of radial profiles of plasma parameters}

The contracted MW discharges for $\mathrm{CO}_{2}$ conversion have been studied in detail in the experimental works in Wolf et al (2019) and Wolf et al (2020b). In Wolf et al (2019), the electron density in the $\mathrm{MW} \mathrm{CO}_{2}$ conversion discharge has been measured line-integrated, and its peak $n_{\mathrm{e} 0}$ has been taken from $n_{\mathrm{e} \Lambda}$ (equation (6)) assuming $s=1$, i.e. that $\Lambda_{n_{\mathrm{e}}}=\Lambda_{777}$. This measurement, along with that of $T_{\mathrm{g}}$ in the plasma core through Doppler broadening, has revealed an ionisation degree in the core $\left(n_{\mathrm{e} 0} / n_{\mathrm{g} 0}\right)$ of the order of $10^{-5}$ in L-mode conditions and rising to $10^{-4}$ in $\mathrm{H}$-mode. Moreover, it has been established in Wolf et al (2019) that the plasma radius $\Lambda_{n_{\mathrm{e}}} / 2$ is of the same order of the skin-depth of MW absorption and that $n_{\mathrm{e} 0} / n_{\mathrm{g} 0}$ is approximately inversely proportional to the square of the plasma diameter: $n_{\mathrm{e} 0} / n_{\mathrm{g} 0} \propto \Lambda_{n_{\mathrm{e}}}^{-2}$. These assertions characterise discharge contracted modes and have been completed by the study in Wolf et al (2020b), where the mechanism of thermo-chemical instability has been proposed to explain the relationship between mode transition and $T_{\mathrm{g}}$. The present study further supplements this body of work, finding that, for the conditions of relevance, $\Lambda_{n_{\mathrm{e}}} \simeq 1.6 \times \Lambda_{777}$ and thus that $n_{\mathrm{e} 0}$ has been previously overestimated by approximately a factor 1.6. This finding allows to correct the calculations from previous works and should be considered in studies of discharge contraction, a phenomenon that depends on electron-impact ionisation and recombination rates. However, the correction does not affect the conclusions from Wolf et al (2019) and Wolf et al (2020b) concerning mode transition and ionisation degree.

The study of these contracted discharges has been complemented by the numerical investigations in Groen et al (2019) and Viegas et al (2020). As mentioned previously, in 
Table 5. Summary of the hypotheses listed in section 2.1 and of the results of the numerical and experimental verifications performed in this work.

\begin{tabular}{lcc}
\hline Nbr & Hypothesis & Verification \\
\hline 1 & $I_{777} \simeq \tau_{\text {rad }}^{-1} \frac{n_{\mathrm{e}}{ }_{\mathrm{O}\left(2 \mathrm{p}^{4}{ }^{3} \mathrm{P}\right)} k_{\mathrm{exc}}\left(T_{\mathrm{e}}\right)}{\tau_{\text {rad }}^{-1}+n_{\mathrm{g}} k_{\mathrm{Q}}}$ & Approximately valid \\
2 & $I_{777} \propto n_{\mathrm{e}} x_{\mathrm{O}} k_{\mathrm{exc}}\left(T_{\mathrm{e}}\right)$ & $I_{777} \propto \tau_{\text {rad }}^{-1} n_{\mathrm{e}} x_{\mathrm{O}} k_{\mathrm{eff}}\left(T_{\mathrm{e}}\right)$ \\
3 & $E / n_{\mathrm{g}}$ and $k_{\mathrm{exc}}$ radially homogeneous & $E / n_{\mathrm{g}}$ concave and $k_{\mathrm{exc}} \propto n_{\mathrm{e}}$ \\
4 & $I_{777} \propto n_{\mathrm{e}}^{s}, 1 \leqslant s \leqslant 2$ & $I_{777} \propto n_{\mathrm{e}}^{s}, 1.82 \leqslant s \leqslant 2.94, s \sim 2.5$ \\
5 & $x_{\mathrm{O}}$ radially homogeneous & Measured $x_{\mathrm{O}}$ concave or homogeneous \\
6 & $I_{777} \propto n_{\mathrm{e}} \propto P_{\mathrm{abs}}$ & $I_{777} \propto P_{\mathrm{abs}}^{s_{\mathrm{p}}}, 1.22 \leqslant s_{\mathrm{p}} \leqslant 2.50, s_{\mathrm{p}} \sim 2.5$ \\
\hline
\end{tabular}

Groen et al (2019) a 3D EM model has simulated the spatial distributions of electric field in these plasmas, given an input profile of $n_{\mathrm{e}}$. The assumption of $s=1$ has been used to determine that profile in both the radial and axial directions. For the case of a low-confinement discharge at 100 mbar with a maximum temperature around $4000 \mathrm{~K}$, a $n_{\mathrm{e}}(r)$ profile with similar FWHM as the corrected $\Lambda_{n_{\mathrm{e}}}$ in condition $\mathrm{A}$ in this work ( $\sim 10 \mathrm{~mm}$ ) but 6 times lower peak, has been considered in Groen et al (2019). The resulting radial profiles of $E$ and $E / n_{\mathrm{g}}$ are rather flat for $r<4 \mathrm{~mm}$, with values almost twice as much as those reported for condition A in this work (figure 8), and have a convex shape radially outwards. Conversely, in this work, $E / n_{\mathrm{g}}$ has a concave radial profile and $E$ is approximately flat up to $r=10 \mathrm{~mm}$. We should notice that different assumptions have been taken on the $T_{\mathrm{g}}$ and $n_{\mathrm{e}}$ profiles and that the method to simulate the electric field is significantly different. While in the plasma reaction-diffusion model of this work $E / n_{\mathrm{g}}(r)$ is found by matching the input $n_{\mathrm{e}}(r)$, in Groen et al (2019) $E$ has been found in the whole domain for a given $n_{\mathrm{e}}$ profile by matching the impedance of the plasma with that of the incoming MW field. The model in Groen et al (2019) finds $E$ by solving Maxwell's equations and by assuming power deposition through Ohmic heating and a homogeneous electron-neutral collision frequency. The finding from this work that $\Lambda_{n_{\mathrm{e}}} \simeq 1.6 \times \Lambda_{777}$ means that the profile of $n_{\mathrm{e}}$ should be even broader than that used in Groen et al (2019). The adoption of a broader profile of $n_{\mathrm{e}}$ in the model of Groen et al (2019) would arguably lead to a broader profile of $E$, i.e. a profile of $E$ rather flat along a region larger than $4 \mathrm{~mm}$ radius. Furthermore, with higher values of $n_{\mathrm{e}}$, it is predicted that lower values of $E$ would be required to maximise the power transfer to the plasma. Finally, for radial positions beyond the plasma, the different ways of calculating the electric field in the different models determine that $E(r)$ increases with $r$ in Groen et al (2019) and decreases with $r$ in this work, which should not be directly compared. Taking these features into account, the profiles of $E(r)$ and $E / n_{\mathrm{g}}(r)$ simulated with the models in Groen et al (2019) and in this work are expected to be coherent with each other.

The numerical study in Viegas et al (2020) using a zero-dimensional model has also taken $n_{\mathrm{e} 0}$ as input under the assumption of $s=1$, mostly in the plasma conditions described in this work as set of conditions C. However, the model in Viegas et al (2020) has not directly used any assumption on $\Lambda_{n_{\mathrm{e}}}$. We should notice that the correction associated to the interferometry measurements described in section
2.2 would lead most of the $n_{\mathrm{e} 0}$ values used in Viegas et al (2020) (those in hybrid regime and in H-mode) to increase by a factor of approximately 1.5. We have also seen in figure 12 that $n_{\mathrm{e} 0}$ should be divided by about 1.6, due to the phenomenon of optical contraction. As a result, the two corrections would lead the input values of $n_{\mathrm{e} 0}$ used in Viegas et al (2020) to stay approximately the same. As such, no significant changes are expected in the simulation results and in the conclusions in that paper. Indeed, despite the different models, the values of peak power density simulated in that work are very similar to the corrected $P_{\mathrm{abs} 0}$ obtained for the set of conditions $\mathrm{C}$ in this work, since the biggest contribution to the calculation of $P_{\mathrm{abs} 0}$ is the local collisional term in equation (18). However, in Viegas et al (2020), the simulated $P_{\mathrm{abs} 0}$ has been compared to the experimentally-estimated one (see figure 3 in Viegas et al (2020)). Experimentally, $P_{\text {abso }}$ in the centre of the plasma has been obtained, as described in Wolf et al (2020b), by normalisation of the power density profile to the total power input. This profile has been assumed the same as the one of emission intensity. If, by following the results of this work, 1.6 times higher diameter and length were assumed for the Gaussian profile of $P_{\text {abs }}$, we could expect $P_{\text {abs } 0}$ to be $1.6^{3} \simeq 4$ times lower. This is a very important difference that appears as the result of the current study. Concerning the comparison of numerical and experimental $P_{\text {abs0 }}$ in figure 3 of Viegas et al (2020), this correction would explain the discrepancies observed and would lead to a significantly better agreement for pressures below 200 mbar, which increases our confidence in the validity of that model.

The two-dimensional model in Wolf et al (2020a), that predicts reactor performances and the mechanisms to obtain them, is also dependent on the spatial profiles of $n_{\mathrm{e}}$ and $P_{\mathrm{abs}}$, as explained in the previous section. As shown in figure 13 , the broadening of these profiles leads to a broader hightemperature profile and increased dissociation in the plasma region. However, a more extended temperature profile also leads to slower cooling downstream and thus a broader recombination region. The result of these two effects is higher conversion and energy efficiencies of the simulated reactor at pressures below 140 mbar but lower performances at higher pressures. The broadening of the $P_{\text {abs }}$ profile in the flow model and the decrease of its peak also has an influence on the peak of the turbulent viscosity $\left(\nu_{\text {T,peak }}\right.$ in equation (16)), that is adjusted to match the peak gas temperature with the one measured in Doppler broadening measurements (Wolf et al 2019). Indeed, lower $\nu_{\text {T,peak }}$ is obtained, which leads to less gas mixing in 


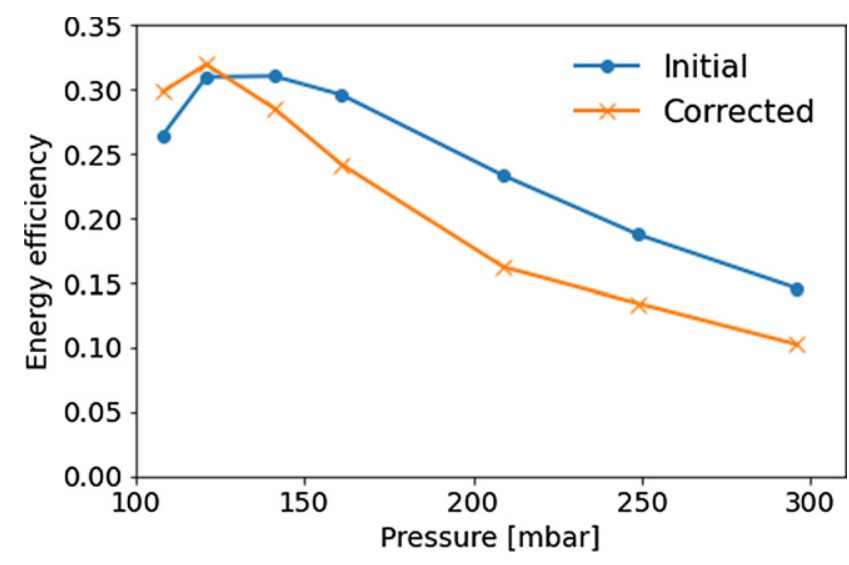

Figure 14. Energy efficiencies as function of pressure, simulated with the flow model in Wolf et al (2020a), using the initial assumptions on $n_{\mathrm{e}}(r)$ and $P_{\mathrm{abs}}(r)\left(\Lambda_{P_{\mathrm{abs}}}=\Lambda_{n_{\mathrm{e}}}=\Lambda 777\right)$, and the corrected $n_{\mathrm{e}}(r)$ and $P_{\text {abs }}(r)$ profiles with $\Lambda_{P_{\text {abs }}} \sim \Lambda_{n_{\mathrm{e}}} \sim 1.6 \times \Lambda_{777}$. Results obtained for the set of conditions $\mathrm{C}$.

the simulated domain. However, the broadening of the hightemperature profile allows for dissociation further from the plasma core and thus the negative effect for dissociation of lower gas mixing becomes negligible. The energy efficiencies simulated with the flow model before and after the correction of the spatial profiles are compared in figure 14 for the 7 cases of the set of conditions $\mathrm{C}$. The increase of these efficiencies at lower pressures improves the agreement of the flow simulations with experimental measurements (figure 6 in Wolf et al (2020a)), as the broader spatial profiles of $P_{\mathrm{abs}}$ and $T_{\mathrm{g}}$ explain the high experimental conversions at low pressure. It is shown that the accurate determination of spatial profiles of discharge parameters should be taken into account when analysing reactor performances. However, for pressures above $140 \mathrm{mbar}$, the agreement between the flow simulations and the measurements does not improve with the correction. This reinforces the statement in Wolf et al (2020a) according to which the energy efficiency at higher pressures depends on the recombination of $\mathrm{CO}$ downstream from the plasma, and thus on the neutral chemistry for temperatures below $2000 \mathrm{~K}$.

Although in this section we have focussed on the implications of spatially-resolved plasma parameters for the study of MW contracted discharges for $\mathrm{CO}_{2}$ conversion, we expect the methods proposed in this work to be relevant for research on other discharges. Firstly, because we have shown that the phenomenon of optical contraction is present not only in atomic plasmas (Golubovskii et al 2011, 2020), but also in a molecular gas like $\mathrm{CO}_{2}$. More generally, we can say that, as spatiallyresolved parameters are often difficult to measure, spontaneous optical emission, a natural phenomenon in non-equilibrium plasmas, can provide valuable insight into the spatial distribution of key plasma parameters when interpreted using spatially resolved CRMs. This is particularly the case in plasma sources where the spatial distribution of optical emission is clearly measurable, such as in steady-state DC, RF and MW discharges in cylindrical containers with radii of a few $\mathrm{mm}$ or a few cm. Another requirement for the application of the proposed approach is the previous knowledge of line-averaged or volume-averaged values of electron density or electric field or power density, allowing to take assumptions on their spatial profiles and then correcting them. Other experimental measurements are not required for the application of the methods proposed, although they may be useful as additional model inputs to guarantee that simulations and experiments assess the same conditions. Following the approach we have employed in this work allows to find spatial profiles of different plasma parameters that are important for both understanding and application of discharges.

\section{Conclusions}

In the present study, a method has been presented to obtain spatially-resolved discharge parameters from emission intensity measurements. Contracted MW discharges for $\mathrm{CO}_{2}$ conversion at intermediate to high pressures (100-300 mbar) have been addressed, where spatial distributions of the Abelinverted intensity of the $777 \mathrm{~nm}$ line emission $\left(\mathrm{O}\left(3 \mathrm{p}{ }^{5} \mathrm{P}\right) \rightarrow\right.$ $\left.\mathrm{O}\left(3 \mathrm{~s}{ }^{5} \mathrm{~S}^{0}\right)\right), I_{777}$, have been measured. Previous experimental studies on these discharges have relied on unverified assumptions on the spatial profiles of discharge parameters, such as electron density $\left(n_{\mathrm{e}}\right)$, power density $\left(P_{\mathrm{abs}}\right)$ and reduced electric field $\left(E / n_{\mathrm{g}}\right)$. These profiles have been based on the Gaussian spatial distribution of $I_{777}$. In this paper, a $1 D$-radial reaction-diffusion plasma model, including a CRM of atomic oxygen, has been developed and used to resolve discharge parameters in space, by matching the measured $I_{777}$ profiles. It is the first time that such a model has been developed in the context of reactive $\mathrm{CO}_{2}$ mixtures and used in different conditions of low-confinement and high-confinement discharges. The simulations have allowed us to verify that the experimentally-assumed profile of $n_{\mathrm{e}}$ is not fully consistent with the measurements of the FWHM of $I_{777}$. Then, they have been used to derive more accurate spatial profiles of $n_{\mathrm{e}}$, $P_{\mathrm{abs}}$ and $E / n_{\mathrm{g}}$, that have been shown to be consistent with the measurements of $I_{777}$. Therefore, we have proposed to use this model to obtain accurate radially-resolved discharge parameters from simulations using experimental input.

The numerically-obtained radial profiles of $n_{\mathrm{e}}$ at intermediate to high pressures follow a Gaussian curve with a lower peak than initially assumed and a higher FWHM than the experimentally-observed $I_{777}$. This phenomenon has been called optical contraction when first reported for atomic plasmas, and has been observed here for the first time in $\mathrm{CO}_{2}$ MW discharges. According to the simulation results, the optical contraction is mostly justified by a concave profile of $E / n_{\mathrm{g}}$, resulting from the self-reinforcing cycle of electron-driven collisionality and gas heating in contracted discharges. This leads to an approximately linear relationship between $n_{\mathrm{e}}$ and the electron-impact excitation coefficient of $\mathrm{O}\left(3 \mathrm{p}{ }^{5} \mathrm{P}\right)$, the upper state of the $777 \mathrm{~nm}$ transition. The optical contraction is also promoted by the concave radial profile of the molar fraction of atomic oxygen in the $\mathrm{CO}_{2}$ conversion plasma, measured through Raman scattering, that in low-confinement conditions is almost linearly proportional to $n_{\mathrm{e}}$. The numerically-obtained radial profile of $P_{\text {abs }}$ is close to the one of $n_{\mathrm{e}}$. The relationships between $P_{\mathrm{abs}}, n_{\mathrm{e}}$ and $I_{777}$ have been shown to be complex and 
dependent on particular conditions. However, we can generally say that for pressures below 150 mbar the FWHM of $P_{\text {abs }}$ and $n_{\mathrm{e}}$ are around 1.6 times higher than that of $I_{777}$. That implies that the peaks of $n_{\mathrm{e}}$ and $P_{\mathrm{abs}}$ are, respectively, around 1.6 and $1.6^{3} \sim 4$ times lower than previously considered.

Finally, the implications of the proposed corrections to the spatial profiles of discharge parameters on the study of contracted MW discharges for $\mathrm{CO}_{2}$ conversion have been demonstrated and discussed. The corrections have an impact on our perception of the discharge diameter and of the electric field and power density distributions in the plasma, as well as on our understanding of the main mechanisms determining reactor performances. Indeed, the broader profile of $P_{\mathrm{abs}}$ than initially supposed explains the high conversion rates measured for pressures below 140 mbar. Moreover, we have proposed that the procedure developed in this work to determine spatial profiles of discharge parameters can be easily used for the study of plasmas with different configurations and different gases. It has been demonstrated that by combining spatiallyresolved numerical simulations with experimental measurements, including measurements of radiative emission intensity, other plasma parameters, such as $n_{\mathrm{e}}, P_{\mathrm{abs}}$ and $E / n_{\mathrm{g}}$, can be retrieved with spatial resolution and replace commonly-used assumptions.

\section{Acknowledgments}

The work presented in this paper is part of the European project KEROGREEN, which has received funding from the European Union's Horizon 2020 Research and Innovation Programme under Grant Agreement No. 763909. This work is also part of the Shell-NWO/FOM initiative 'Computational sciences for energy research' of Shell and Chemical Sciences, Earth and Life Sciences, Physical Sciences, FOM and STW. This research has also been carried out under TTW open technology project (Grant No. 15325) in collaboration with Gasunie, Stedin, DNVGL and Ampleon. The work of AvdS received funding from the Netherlands Organization for Scientific Research (NWO) in the framework of the $\mathrm{CO}_{2}$ to-Products programme with kind support from Shell, and the ENW PPP Fund for the top sectors. We thank Prof. Savino Longo from the University of Bari for discussions on the Monte Carlo Flux method and Prof. Jan van Dijk from the Eindhoven University of Technology for suggestions on fluid modelling. We are also thankful to Tim Righart from DIFFER for gas temperature measurements and to Pieter Willem Groen from DIFFER for discussions on MW power absorption.

\section{Data availability statement}

The data that support the findings of this study are available upon reasonable request from the authors.

\section{ORCID iDs}

P Viegas (1) https://orcid.org/0000-0002-3820-3300

L Vialetto (i) https://orcid.org/0000-0003-3802-8001

A W van de Steeg (D) https://orcid.org/0000-0002-2976-7905

G J van Rooij (D) https://orcid.org/0000-0003-4795-3274

M C M van de Sanden (D) https://orcid.org/0000-0002-41199971

P Diomede (iD https://orcid.org/0000-0002-4523-3049

F J J Peeters (iD https://orcid.org/0000-0002-7759-2935

\section{References}

Alves L L, Bogaerts A, Guerra V and Turner M M 2018 Plasma Sources Sci. Technol. 27023002

Alves L L, Coche P, Ridenti M A and Guerra V 2016 Eur. Phys. J. D 70124

Annušová A, Marinov D, Booth J P, Sirse N, da Silva M L, Lopez B and Guerra V 2018 Plasma Sources Sci. Technol. 27045006

Baeva M, Hempel F, Baierl H, Trautvetter T, Foest R and Loffhagen D 2018 J. Phys. D: Appl. Phys. $\mathbf{5 1} 385202$

Barklem P S 2007 Astron. Astrophys. 462 781-8

Bongers W et al 2017 Plasma Process. Polym. 14 e1600126

Butylkin Y P, Zhivotov V K, Krasheninnikov E G, Krotov M F, Rusanov V D, Tarasov Y V and Fridman A A 1981 Sov. Phys. Tech. Phys. $26555-8$

Capitelli M, Bruno D and Laricchiuta A 2013 Fundamental Aspects of Plasma Chemical Physics: Transport (New York: Springer)

Capitelli M, Celiberto R, Colonna G, Esposito F, Gorse C, Hassouni K, Laricchiuta A and Longo S 2015 Fundamental Aspects of Plasma Chemical Physics: Kinetics vol 85 (Springer)

Caplinger J E and Perram G P 2020 Plasma Sources Sci. Technol. 29 015011

Carbone E A D, Hübner S, Palomares J M and van der Mullen J J A M 2012 J. Phys. D: Appl. Phys. 45345203

D'Isa F A, Carbone E A D, Hecimovic A and Fantz U 2020 Plasma Sources Sci. Technol. 29105009

Dagdigian P J, Forch B E and Miziolek A W 1988 Chem. Phys. Lett. 148 299-308

den Harder N et al 2017 Plasma Process. Polym. 14 e1600120

Durandet A, Arnal Y, Margot-Chaker J and Moisan M 1989 J. Phys. D: Appl. Phys. 221288

Dyatko N A, Ionikh Y Z, Kochetov I V, Marinov D L, Meshchanov A V, Napartovich A P, Petrov F B and Starostin S A 2008 J. Phys. D: Appl. Phys. 41055204

Eliasson B and Kogelschatz U 1986 J. Phys. B: At. Mol. Phys. 19 1241-7

Erdman P W and Zipf E C 1987 J. Chem. Phys. 874540

Fiebrandt M, Bibinov N and Awakowicz P 2020 Plasma Sources Sci. Technol. 29045018

Fiebrandt M, Hillebrand B, Spiekermeier S, Bibinov N, Böke M and Awakowicz P 2017 J. Phys. D: Appl. Phys. 50355202

Fleisch T, Kabouzi Y, Moisan M, Pollak J, Castaños-Martínez E, Nowakowska H and Zakrzewski Z 2007 Plasma Sources Sci. Technol. 16 173-82

Fridman A 2008 Plasma Chemistry (Cambridge: Cambridge University Press)

Fridman A and Kennedy L A 2004 Plasma Physics and Engineering (London: Taylor and Francis)

Georgieva V et al 2017 Plasma Process. Polym. 141600185

Giovangigli V 1990 Impact Comput. Sci. Eng. 2 73-97

Goede A P H, Bongers W A, Graswinckel M F, van de Sanden R M C M, Leins M, Kopecki J, Schulz A and Walker M 2014 EPJ Web Conf. 7901005 
Golubovskii Y B, Kalanov D and Maiorov V 2017 Phys. Rev. E 96 023206

Golubovskii Y B, Nekuchaev V, Gorchakov S and Uhrlandt D 2011 Plasma Sources Sci. Technol. 20053002

Golubovskii Y B, Siasko A V and Nekuchaev V O 2020 Plasma Sources Sci. Technol. 29065020

Gordiets B F, Ferreira C M, Guerra V L, Loureiro J M A H, Nahorny J, Pagnon D, Touzeau M and Vialle M 1995 IEEE Trans. Plasma Sci. 23 750-68

Gregório J, Boisse-Laporte C and Alves L L 2010 Eur. Phys. J. Appl. Phys. 4913102

Gregório J, Leprince P, Boisse-Laporte C and Alves L L 2012 Plasma Sources Sci. Technol. 21015013

Groen P W C, Wolf A J, Righart T W H, van de Sanden M C M, Peeters F J J and Bongers W A 2019 Plasma Sources Sci. Technol. 28075016

Grofulović M, Alves L L and Guerra V 2016 J. Phys. D: Appl. Phys. 49395207

Guerra V, Silva T, Ogloblina P, Grofulović M, Terraz L, da Silva M L, Pintassilgo C D, Alves L L and Guaitella O 2017 Plasma Sources Sci. Technol. 26 11LT01

Hirschfelder J O and Curtiss C F 1949 Flame propagation in explosive gas mixtures 3rd Symp. on Combustion, Flame and Explosion Phenomena pp 121-7

Ikegami H 1968 Japan. J. Appl. Phys. 7 634-55

Itikawa Y 2009 J. Phys. Chem. Ref. Data 381

Jimenez-Diaz M, Carbone E A D, van Dijk J and van der Mullen J J A M 2012 J. Phys. D: Appl. Phys. 45335204

Kabouzi Y, Graves D B, Martinez E C and Moisan M 2007 Phys. Rev. E 75016402

Kanik I, Noren C, Makarov O P, Vattipalle P, Ajello J M and Shemansky D E 2003 J. Geophys. Res. 1085126

Koelman P, Heijkers S, Tadayon Mousavi S, Graef W, Mihailova D, Kozák T, Bogaerts A and van Dijk J 2017 Plasma Process. Polym. 141600155

Kossyi I A, Kostinsky A Y, Matveyev A A and Silakov V P 1992 Plasma Sources Sci. Technol. 1 207-20

Kozák T and Bogaerts A 2014 Plasma Sources Sci. Technol. 23 045004

Kramida A, Ralchenko Y, Reader J and Team N A 2020 NIST Standard Reference Database 78-Atomic Spectra Database (Ver. 5.8) GaithersburgNational Institute of Standards and Technology

Laher R R and Gilmore F R 1990 J. Phys. Chem. Ref. Data 19 277

Laricchiuta A et al 2009 Eur. Phys. J. D 54 607-12

Lawrence G M 1970 Phys. Rev. A 2 387-407

Lieberman M A and Lichtenberg A J 2005 Principles of Plasma Discharges and Materials Processing (New York: Wiley)

Martinez E C, Kabouzi Y, Makasheva K and Moisan M 2004 Phys. Rev. E 70066405

McConkey J W, Malone C P, Johnson P V, Winstead C, McKoy V and Kanik I 2008 Phys. Rep. 466 1-103

Mewe R 1967 Br. J. Appl. Phys. 18 107-18

Moisan M and Pelletier J 2012 Physics of Collisional Plasmas: Introduction to High-Frequency Discharges (Springer)

Mori T, Kanou K, Mizuta K, Kuramasu T, Ishikawa Y and Arai S 1992 J. Chem. Phys. 979094

Morillo-Candas A S, Drag C, Booth J-P, Dias T C, Guerra V and Guaitella O 2019 Plasma Sources Sci. Technol. 28075010

Mousavi S T, Carbone E, Wolf A, Bongers W and van Dijk J 2021 Two-temperature balance equations implementation, numerical validation and application to $\mathrm{H}_{2} \mathrm{O}-\mathrm{He}$ microwave induced plasmas https://doi.org/10.1088/1361-6595/ac0a44
Niemi K, von der Gathen V S and Döbele H F 2001 J. Phys. D: Appl. Phys. 34 2330-5

Parker J V 1963 Phys. Fluids 6 1657-8

Patankar S V 1980 Numerical Heat Transfer and Fluid Flow (London: Taylor and Francis)

Petrov G M and Ferreira C M 1999 Phys. Rev. E 59 3571-82

Pietanza L D, Colonna G and Capitelli M 2020 Plasma Sources Sci. Technol. 29035022

Ridenti M A, Amorim J D, Pino A D, Guerra V and Petrov G 2018 Phys. Rev. E 97013201

Santos M, Noël C, Belmonte T and Alves L L 2014 J. Phys. D: Appl. Phys. 47265201

Schaefer G and Hui P 1990 J. Comput. Phys. 89 1-30

Schottky W 1924 Phys. Z. 25342

Shneider M N, Mokrov M S and Milikh G M 2014 Phys. Plasmas 21 032122

Silva A F, Morillo-Candás A S, Tejero-del-Caz A, Alves L L, Guaitella O and Guerra V 2020 Plasma Sources Sci. Technol. 29125020

Smith G P et al 2018 GRI-MECH 3.0 database http://combustion .berkeley.edu/gri-mech/version30/text30.html

Snoeckx R and Bogaerts A 2017 Chem. Soc. Rev. 46 5805-63

Stancu G D, Leroy O, Coche P, Gadonna K, Guerra V, Minea T and Alves L L 2016 J. Phys. D: Appl. Phys. 49435202

Sushkov V P, Do H T and Hippler R 2013 Contrib. Plasma Phys. 53 549-59

Synek P, Obrusník A, Hübner S, Nijdam S and Zajíčková L 2015 Plasma Sources Sci. Technol. 24025030

van de Steeg A W, Butterworth T, van den Bekerom D C M, Silva A F, van de Sanden M C M and van Rooij G J 2020 Plasma Sources Sci. Technol. 29115001

van de Steeg A W, Vialetto L, Silva A F, Peeters F J J, van den Bekerom D C M, Gatti N, Diomede P, van de Sanden M C M and van Rooij G J 2021 Opt. Lett. 46 2172-5

van den Bekerom $\mathrm{D}$ C $\mathrm{M}$, Linares $\mathrm{J} \mathrm{M}$ P, Verreycken $\mathrm{T}$, van Veldhuizen E M, Nijdam S, Berden G, Bongers W A, van de Sanden M C M and van Rooij G J 2019 Plasma Sources Sci. Technol. 28055015

van den Bekerom D C M, van de Steeg A, van de Sanden M C M and van Rooij G J 2020 J. Phys. D: Appl. Phys. 53054002

van Gessel A F H, Carbone E A D, Bruggeman P J and van der Mullen J J A M 2012 Plasma Sources Sci. Technol. 21 015003

van Rooij G J et al 2015 Faraday Discuss. 183 233-48

Vermeiren V and Bogaerts A 2020 J. Phys. Chem. C 124 18401-15

Vialetto L, Longo S and Diomede P 2019 Plasma Sources Sci. Technol. 28115015

Vialetto L, Viegas P, Longo S and Diomede P 2020 Plasma Sources Sci. Technol. 29115006

Viegas P, Vialetto L, Wolf A J, Peeters F J J, Groen P W C, Righart T W H, Bongers W A, van de Sanden M C M and Diomede P 2020 Plasma Sources Sci. Technol. 29105014

Wolf A J, Peeters F J J, Groen P W C, Bongers W A and van de Sanden M C M 2020a J. Phys. Chem. C 124 16806-19

Wolf A J, Righart T W H, Peeters F J J, Bongers W A and van de Sanden M C M 2020b Plasma Sources Sci. Technol. 29 025005

Wolf A J, Righart T W H, Peeters F J J, Groen P W C, van de Sanden M C M and Bongers W A 2019 Plasma Sources Sci. Technol. 28115022

Yimer I, Campbell I and Jiang L-Y 2002 Can. Aeronaut. Space J. 48 195-200

Zhong H, Shneider M N, Mokrov M S and Ju Y 2019 J. Phys. D: Appl. Phys. 52484001 